\title{
ELEMENTS OF LIPSCHITZ TOPOLOGY
}

\author{
J. LUUKKAINEN and J. VÄISÄLÄ
}

\section{Contents}

1. Introduction 85

2. Basic properties of Lipschitz and LIP maps 87

3. Lipschitz manifolds 97

4. Embedding 102

5. Extension and approximation 105

6. General position 109

7. Collaring and the Schönflies problem 113

8. LIP structures of $R^{n}, S^{n}$, and $I^{n} 117$

9. Open problems 120

References 121

\section{Introduction}

1.1. A map $f$ of a metric space $(X, d)$ into a metric space $\left(Y, d^{\prime}\right)$ is said to be a Lipschitz map if there is a constant $L$ such that

$$
d^{\prime}(f(x), f(y)) \leqq L d(x, y)
$$

for all $x, y$ in $X$. If every $x \in X$ has a neighborhood $U$ such that $f \mid U$ is Lipschitz, $f$ is said to be locally Lipschitz (abbreviated LIP). If $X$ is compact, every LIP map of $X$ is Lipschitz. We also let LIP denote the category of metric spaces and LIP maps. An isomorphism in the category LIP is called a lipeomorphism. Thus a lipeomorphism is a bijective map $f$ such that both $f$ and $f^{-1}$ are LIP.

Lipschitz topology can be defined as the study of those properties of metric spaces which are invariant under lipeomorphisms. We shall be particularly interested in Lipschitz manifolds: metric spaces which are locally lipeomorphic to a euclidean space.

Let us compare LIP with three important categories: TOP = topological spaces and continuous maps, PL = polyhedra and PL maps, and DIFF = smooth manifolds and smooth maps. Without an essential loss of generality, we may assume 
that each polyhedron and each smooth manifold is embedded in a euclidean space. Then they are metric spaces, and PL and smooth maps are LIP. Thus we have the diagram

$$
\begin{aligned}
& \mathrm{DIFF} \\
& \mathrm{PL}
\end{aligned}
$$

where each arrow is a forgetful functor. Alternatively, we could use locally metric spaces, see 3.4 .

There is an extensive literature on the topology of manifolds in the categories TOP, PL, and DIFF. Lipschitz manifolds have been mentioned in some papers, especially in Whitehead [31], but no systematic treatment seems to be published so far.

In this paper we give the basic geometric tools needed in Lipschitz topology. These include the cone construction, extension and approximation of maps, general position and collaring. We also give some results concerning LIP embedding, the Schönflies problem, and the LIP Hauptvermutung. For example, we show that for $n \neq 4, R^{n}$ has a unique LIP structure.

1.3. Notation and terminology. Our set-theoretical and topological notations are fairly standard. We let $A \backslash B$ denote the set-theoretical difference of $A$ and $B$, and $\mathbf{C} A$ is the complement of $A$ in a given space. A singleton $\{x\}$ is usually written simply as $x$. The symbols $\partial A$ and int $A$ are used, somewhat ambiguously, for both manifolds and subspaces. A map is always continuous, a function need not be.

We let $R^{n}$ denote the euclidean $n$-space, and $R_{+}^{n}$ is the closed upper half space $\left\{x \in R^{n} \mid x_{n} \geqq 0\right\}$. The standard orthogonal basis for $R^{n}$ is written as $\left(e_{1}, \ldots, e_{n}\right)$. If $p<n$, we identify $R^{p}$ with the subspace $R^{p} \times 0$ of $R^{n}$ by $\left(x_{1}, \ldots, x_{p}\right)=$ $=\left(x_{1}, \ldots, x_{p}, 0, \ldots, 0\right)$. We shall use the euclidean norm $|x|=\left(x_{1}^{2}+\ldots+x_{n}^{2}\right)^{1 / 2}$ and the euclidean distance $d(x, y)=|x-y|$ in $R^{n}$. Given two sets $A, B$ in $R^{n}, A B$ is their rectilinear join. Given two vectors $x, y$ in $R^{n}$, we let $x \cdot y$ denote their inner product, and

$$
\operatorname{ang}(x, y)=\arccos \frac{x \cdot y}{|x||y|}, \quad \operatorname{ac}(x, y)=\arccos \frac{|x \cdot y|}{|x||y|}
$$

are the angle and the acute angle between $x$ and $y$, with the special conventions ang $(x, y)=\pi$, ac $(x, y)=\pi / 2$, if $x=0$ or $y=0$.

When we are dealing with a metric space $X$, the letter $d$ will stand for the metric of $X$. If we consider maps of $X$ into another space $Y$, we shall use $d^{\prime}$ for the metric of $Y$. The distance between two non-empty sets $A, B$ in $X$ will be written as $d(A, B)$, and the diameter of $A$ by $d(A)$ with $d(\emptyset)=0$. If $S$ is a set and if $f, g: S \rightarrow X$ are functions, then $d(f, g)=\sup \{d(f(x), g(x)) \mid x \in S\}$ is the distance between $f$ and $g$. For $a \in X$ and $r>0$, we let $B(a, r)$ denote the open ball $\{x \mid d(x, a)<r\}$. If $X=R^{n}$, we may use the notation $B^{n}(a, r)$ for $B(a, r)$ and the abbreviations $B^{n}(r)=B^{n}(0, r)$, $B^{n}=B^{n}(1)$. For spheres, we write $S^{n-1}(a, r)=\partial B^{n}(a, r)=\left\{x \in R^{n}|| x-a \mid=r\right\}, S^{n-1}(r)=$ 
$=S^{n-1}(0, r)$, and $S^{n-1}=S^{n-1}(1)$. The unit cube in $R^{n}$ is $I^{n}=[-1,1]^{n}$. Thus $I^{1}$ is different from the standard interval $I=[0,1]$. We shall also use the symbol $I$ for a general indexing set if there is no danger of misunderstanding. $\mathbb{Z}$ is the set of integers and $\mathbf{N}$ is the set of positive integers.

The definitions for a Lipschitz map, a LIP map (locally Lipschitz map) and a lipeomorphism were given in 1.1. The Lipschitz constant lip $f$ of a Lipschitz map $f: X \rightarrow Y$ is the smallest number $L \geqq 0$ satisfying the condition (1.2). If $\operatorname{lip} f \leqq L, f$ is said to be L-Lipschitz. If $f$ is bijective and if both $f$ and $f^{-1}$ are L-Lipschitz, $f$ is an L-lipeomorphism. If this is true for some $L \geqq 0, f$ is a strong lipeomorphism. If $f: X \rightarrow Y$ is injective and $f$ defines a lipeomorphism $f_{1}: X \rightarrow f X, f$ is a LIP embedding. If $f_{1}$ is a strong lipeomorphism, $f$ is a Lipschitz embedding. If every $x$ in $X$ has a neighborhood $U$ such that $f \mid U$ is a LIP embedding, $f$ is a LIP immersion. Two metrics $d, d^{\prime}$ in a space $X$ are LIP equivalent or Lipschitz equivalent if id: $(X, d) \rightarrow$ $\rightarrow\left(X, d^{\prime}\right)$ is a lipeomorphism or a strong lipeomorphism, respectively.

We remark that Whitehead [31] has used the term "Lipschitz map" for a LIP map and the term "regular Lipschitz map" for a LIP immersion.

\section{Basic properties of Lipschitz and LIP maps}

2.1. We begin by stating some algebraic properties for the class of Lipschitz maps. The straightforward proofs will be omitted.

2.2. Lemma. Suppose that $f: X \rightarrow Y$ and $g: Y \rightarrow Z$ are maps between metric spaces. If $f$ is $L_{1}$-Lipschitz and $g$ is $L_{2}$-Lipschitz, then $g f$ is $L_{1} L_{2}$-Lipschitz. If $f$ and $g$ are LIP, then $g f$ is LIP.

2.3. Lemma. Let $X$ be a metric space, let $f, g: X \rightarrow R^{n}$ and $\varphi, \psi: X \rightarrow R^{1}$ be Lipschitz, and let $c \in R^{1}$. Then $f+g, c f,|f|, \max (\varphi, \psi)$, and $\min (\varphi, \psi)$ are Lipschitz. If $f$ and $\varphi$ are bounded, then $\varphi f$ is Lipschitz. If, in addition, $\varphi$ is bounded away from zero, then $f / \varphi$ is Lipschitz.

2.4. Corollary. Let $X$ be a metric space, let $f, g: X \rightarrow R^{n}$ and $\varphi, \psi: X \rightarrow R^{1}$ be LIP, and let $c \in R^{1}$. Then $f+g, c f,|f|, \max (\varphi, \psi), \min (\varphi, \psi)$, and $\varphi f$ are LIP. If $\varphi(x) \neq 0$ for all $x$, then $f / \varphi$ is LIP.

2.5. Lemma. Let $X$ be a metric space and let $\emptyset \neq A \subset X$. Then the map $x \mapsto d(x, A)$ is 1-Lipschitz. If $A$ and $B$ are disjoint closed sets in $X$, there is a LIP map $f: X \rightarrow[0,1]$ such that $A=f^{-1}(0), B=f^{-1}(1)$.

Proof. The first assertion is elementary and well-known (but extremely useful). To prove the second assertion, we may set $f(x)=d(x, A) /(d(x, A)+d(x, B))$ if $A \neq \emptyset \neq B$. If $B=\emptyset$ and $\emptyset \neq A \neq X$, choose $y \in X \backslash A$ and set $f(x)=d(x, A) / 2(d(x, A)+$ $+d(x, y))$. If $B=\emptyset$ and $A=X$, set $f(x)=0$ for all $x$. If $A=B=\emptyset$, set $f(x)=1 / 2$ for all $x$. The case where $A=\emptyset \neq B$ is treated similarly. 
2.6. Cone construction. Let $a \in R^{n}$ and $Q \subset R^{n}$ be such that $a$ and $Q$ are independent, or equivalently, $a Q$ is a cone. This means that $a \notin Q$ and no ray from $a$ meets $Q$ in more than one point. Each point $x \in a Q$ can be written as $x=\lambda a+\mu y$ with $y \in Q, 0 \leqq \lambda \leqq 1, \lambda+\mu=1$. This representation is unique for $x \neq a$. Given a map $f: Q \rightarrow R^{p}$ and a point $b \in R^{p}$, the $b$-cone of $f$ with vertex $a$ is the function $\bar{f}: a Q \rightarrow R^{p}$ defined by $\bar{f}(\lambda a+\mu y)=\lambda b+\mu f(y)$. In general, $\bar{f}$ need not be continuous. However, if $Q$ is compact, then $\bar{f}$ is continuous. This is one reason why cones work best for compact sets. Observe also that a cone of a compact set is always compact, while a cone of a locally compact set is not usually locally compact at the vertex $a$. Even if $Q$ is compact, a cone of a Lipschitz map $f: Q \rightarrow R^{p}$ need not be Lipschitz. For example, let $Q=\left\{(x, y) \mid 0 \leqq x \leqq 1, y=x^{2}\right\} \subset R^{2}, a=(1,0), b=0 \in R^{1}$, and $f: Q \rightarrow R^{1}$ the constant map $f(x, y)=1$. Then for $z_{1}=(\varepsilon, 0)$ and $z_{2}=\left(\varepsilon, \varepsilon^{2}\right)$ we have $\mid \bar{f}\left(z_{1}\right)-$ $-\bar{f}\left(z_{2}\right)|/| z_{1}-z_{2} \mid=1 / \varepsilon$, and hence $\bar{f}$ is not Lipschitz.

2.7. Definition. Let $Q \subset R^{n}$ be compact, and let $a \in R^{n}$ be such that $a Q$ is a cone. We say that $a$ and $Q$ are Lipschitz independent and $a Q$ is a Lipschitz cone if for every Lipschitz map $f: Q \rightarrow R^{p}$ and for every $b \in R^{p}$, the $b$-cone $\bar{f}: a Q \rightarrow R^{p}$ of $f$ is Lipschitz.

2.8. Radial projection. Let $a \in R^{n}$, and let $Q_{1}, Q_{2} \subset R^{n} \backslash a$. Suppose that each ray from $a$ through $Q_{1}$ meets $Q_{2}$ in exactly one point. Then the radial projection from $Q_{1}$ into $Q_{2}$ with center $a$ is the unique function $f: Q_{1} \rightarrow Q_{2}$ such that $f(x)$ belongs to the ray from $a$ through $x$. If $Q_{2}$ is compact, then $f$ is continuous but not necessarily LIP.

2.9. We are going to establish several equivalent conditions for Lipschitz independence. We first introduce some notation. If $a \in R^{n}, Q \subset R^{n} \backslash a, b \in Q$, we set

$$
\beta(Q, a, b)=\lim _{r \rightarrow 0} \inf \left\{\operatorname{ac}(b-a, x-y) \mid x, y \in Q \cap B^{n}(b, r)\right\} .
$$

It is easy to see that

$$
\beta(Q, a, b)=\lim _{r \rightarrow 0} \inf \left\{\operatorname{ac}(x-a, x-y) \mid x, y \in Q \cap B^{n}(b, r)\right\} .
$$

For $x, y \in R^{n}$ we set $s(x, y)=\{\lambda x+\mu y \mid \lambda+\mu=1, \lambda \mu \leqq 0\}$. If $x=y$, then $s(x, y)=\{x\}$. If $x \neq y$, then $s(x, y)$ is the line through $x$ and $y$ less the open segment between $x$ and $y$. For a set $Q \subset R^{n}$ we write $s(Q)=\cup\{s(x, y) \mid x \in Q, y \in Q\}$.

2.12. Lemma. Let $p: R^{n} \backslash 0 \rightarrow S^{n-1}$ be defined by $p(x)=x /|x|$. Then $|p(x)-p(y)|^{2} \leqq|x-y|^{2} /|x||y|$ and $|p(x)-p(y)||y| \leqq 2|x-y|$ for all $x$ and $y$. Hence $p$ is LIP.

Proof. Using elementary estimates, we obtain $|x||y||p(x)-p(y)|^{2}=2|x||y|-$ $-2 x \cdot y \leqq|x|^{2}+|y|^{2}-2 x \cdot y=|x-y|^{2}$ and $|p(x)-p(y)||y|=|y-| y|x||x||\leqq| y-x \mid+$
+||$x|-| y|| \leqq 2|x-y|$. 
2.13. Theorem. Let $a Q$ be a cone in $R^{n}$ with $Q$ compact. Then the following conditions are equivalent:

(1) $a Q$ is a Lipschitz cone.

(2) If $c: Q \rightarrow R^{1}$ is the constant map $c(x)=1$, the 0 -cone $\bar{c}: a Q \rightarrow R^{1}$ of $c$. is Lipschitz.

(3) $a \notin \overline{s(Q)}$.

(4) $\inf \{\beta(Q, a, b) \mid b \in Q\}>0$.

(5) $\beta(Q, a, b)>0$ for all $b \in Q$.

(6) If $p: R^{n} \backslash a \rightarrow S^{n-1}$ is the map $p(x)=(x-a) /|x-a|, p \mid Q$ is a Lipschitz embedding.

(7) For every set $Q_{1} \subset R^{n}$ such that $a \notin \bar{Q}_{1}$ and such that each ray from a through $Q_{1}$ meets $Q$ in exactly one point, the radial projection $f: Q_{1} \rightarrow Q$ is Lipschitz.

Proof. We shall prove the implications (1) $\Rightarrow(2) \Rightarrow(3) \Rightarrow(4) \Rightarrow(5) \Rightarrow(6) \Rightarrow(1)$ and $(6) \Rightarrow(7) \Rightarrow(6)$. We may assume that $a=0$. Choose positive numbers $r$ and $R$ such that $Q \subset B^{n}(R) \backslash B^{n}(r)$.

$(1) \Rightarrow(2)$ : Trivial.

$(2) \Rightarrow(3)$ : If (3) is false, there are points $x, y \in Q$ and $z \in s(x, y)$ with $|z|=$ $d(0, s(x, y))<(L+1 / r)^{-1}$ where $L=\operatorname{lip} \bar{c}$. Now $|z|<r$ implies $x \neq z \neq y$. Hence $z$ and $x-y$ are orthogonal. We may assume $|z-x|<|z-y|$. Letting $u$ be the orthogonal projection of $x$ on $y$, we obtain

$$
\frac{|\bar{c}(x)-\bar{c}(u)|}{|x-u|}=\frac{|y|-|u|}{|y||x-u|}=\frac{|y-z|}{|y||z|} \geqq \frac{1}{|z|}-\frac{1}{|y|}>L,
$$

which is a contradiction.

$(3) \Rightarrow(4)$ : If (4) is false, it follows from (2.11) that there are sequences of points $x_{j} \in Q, \quad y_{j} \in Q$ such that $0<\left|x_{j}-y_{j}\right| \rightarrow 0$ and $\alpha_{j}=\operatorname{ac}\left(x_{j}, x_{j}-y_{j}\right) \rightarrow 0$. For large $j$ we have $\left|x_{j}-y_{j}\right|<r / \sqrt{2}$ and $\alpha_{j}<\pi / 4$. Then $\left|x_{j}-y_{j}\right|<\left|x_{j}\right| \cos \alpha_{j}$, which implies $d\left(0, s\left(x_{j}, y_{j}\right)\right)=\left|x_{j}\right| \sin \alpha_{j} \leqq R \sin \alpha_{j} \rightarrow 0$. Hence $0 \in \overline{s(Q)}$.

(4) $\Rightarrow(5)$ : Trivial.

$(5) \Rightarrow(6)$ : By 2.12, $p \mid Q$ is Lipschitz. We must show that the inverse $q$ : $p Q \rightarrow Q$ of $p \mid Q$ is LIP. Let $z \in p Q$. Then there is an open neighborhood $U$ of $q(z)$ in $Q$ and a positive number $\beta$ such that ac $(x, x-y)>\beta$ for all $x, y \in U$. Since $Q \backslash U$ is compact, the set $V=p U=p Q \backslash p[Q \backslash U]$ is an open neighborhood of $z$ in $p Q$. If $x, y \in V$ and $\alpha=\operatorname{ac}(q(x), q(x)-q(y))$, then

Hence $q$ is LIP.

$$
|q(x)-q(y)| \leqq \frac{|q(y)|}{\sin \alpha}|x-y| \leqq \frac{R}{\sin \beta}|x-y| .
$$

$(6) \Rightarrow(1)$ : Let $f: Q \rightarrow R^{p}$ be Lipschitz, let $b \in R^{p}$, and let $\bar{f}: a Q \rightarrow R^{p}$ be the $b$-cone of $f$. We must show that $\bar{f}$ is Lipschitz. We may assume that $b=0$. Let $q: p Q \rightarrow Q$ be the inverse of $p \mid Q$. Then $g=f q: p Q \rightarrow R^{p}$ is Lipschitz. Letting $\bar{g}$ 
and $\bar{p}$ denote the 0 -cones of $g$ and $p \mid Q$, respectively, with vertex 0 , we have $\bar{f}=\bar{g} \bar{p}$. Hence it is sufficient to show that $\bar{p}$ and $\bar{g}$ are Lipschitz. Choose $L>0$ and $M>0$ such that $q$ and $g$ are $L$-Lipschitz and $|f(z)| \leqq M$ for all $z \in Q$. For $x, y \in 0 Q \backslash 0$ we obtain

$$
\begin{aligned}
|\bar{p}(x)-\bar{p}(y)| & =\frac{|| q(p(y))|x-| q(p(x))|y|}{|q(p(x))||q(p(y))|} \\
& \leqq \frac{|q(p(y))||x-y|}{r^{2}}+\frac{|| q(p(y))|-| q(p(x))|||y|}{r^{2}} \\
& \leqq \frac{R}{r^{2}}|x-y|+\frac{L}{r^{2}}|p(x)-p(y)||y| .
\end{aligned}
$$

By 2.12, this implies $|\bar{p}(x)-\bar{p}(y)| \leqq(R+2 L) r^{-2}|x-y|$. Since $\bar{p}$ is continuous, this also holds for $x=0$. Hence $\bar{p}$ is Lipschitz.

To show that $\bar{g}$ is Lipschitz let $x, y \in 0(p Q)$. If $x \neq 0 \neq y$, we obtain $|\bar{g}(x)-\bar{g}(y)|=$ $=|| x|g(p(x))-| y|g(p(y))| \leqq|x||g(p(x))-g(p(y))|+|x-y||g(p(y))|$. By 2.12, this implies $|\bar{g}(x)-\bar{g}(y)| \leqq(2 L+M)|x-y|$. Since $\bar{g}$ is continuous, this also holds for $x=0$. Hence $\bar{g}$ is Lipschitz.

$(6) \Rightarrow(7)$ : The radial projection $f: Q_{1} \rightarrow Q$ can be written as $f=q\left(p \mid Q_{1}\right)$ where $p$ is as in (6) and $q: p Q \rightarrow Q$ is the inverse of $p \mid Q$. By 2.12, $p \mid Q_{1}$ is Lipschitz. Hence $f$ is Lipschitz. tion.

(7) $\Rightarrow(6)$ : Trivial, since the inverse $q: p Q \rightarrow Q$ of $p \mid Q$ is a radial projec-

2.14. Corollary. If $a Q$ is a Lipschitz cone and $Q_{1} \subset Q$ is compact, then $a Q_{1}$ is a Lipschitz cone.

Proof: This follows, for example, from (2) of 2.13.

2.15. Extended cones. If $a Q$ is a cone in $R^{n}$, the corresponding extended cone $a Q \infty$ is defined as $\{\lambda a+\mu x \mid x \in Q, \lambda+\mu=1, \mu \geqq 0\}$. It consists of all rays from $a$ through $Q$. If $f: Q \rightarrow R^{p}$ is a map and if $b \in R^{p}$, the extended $b$-cone of $j$ with vertex $a$ is the function $f^{*}: a Q \infty \rightarrow R^{p}$ defined by $f^{*}(\lambda a+\mu x)=\lambda b+\mu f(x)$.

2.16. Theorem. If aQ is a Lipschitz cone, then every extended cone of every Lipschitz map $f: Q \rightarrow R^{p}$ is Lipschitz.

Proof. We may assume that $a=0$ and $f^{*}(a)=0$. Since $f^{*} \mid a Q$ is the cone of $f$, it is $L$-Lipschitz for some $L$. Let $x, y \in a Q_{\infty}$. Choosing $t>0$ so that $t x$ and ty lie in $a Q$, we obtain $\left|f^{*}(x)-f^{*}(y)\right|=\left|t^{-1} f^{*}(t x)-t^{-1} f^{*}(t y)\right| \leqq L|x-y|$.

2.17. Theorem. Suppose that $A \subset R^{n}$ is a compact convex set and that $a \in \operatorname{int} A$. Then $a(\partial A)$ is a Lipschitz cone. 
Proof. Choose $r>0$ such that $B^{n}(a, r) \subset A$. Using the notation of 2.9 we see that $s(x, y) \cap B^{n}(a, r)=\emptyset$ for all $x, y \in \partial A$. The theorem follows from 2.13 (3). $\square$ The result is well-known [16].

2.18. Theorem. Let $P \subset R^{n}$ be a polyhedron. Then every PL map $f: P \rightarrow R^{p}$ is LIP.

Proof. The theorem is obviously true if $\operatorname{dim} P=0$, and we proceed by induction on $\operatorname{dim} P$. Assume that the theorem is true for $\operatorname{dim} P<m$ and suppose $\operatorname{dim} P=m$. Let $a \in P$. Then there is an $n$-cube $A$, centered at $a$, such that $P \cap \partial A=Q$ is compact, $P \cap A=a Q$, and $f \mid P \cap A$ is a cone of $f \mid Q$. Since $Q$ is a polyhedron with $\operatorname{dim} Q<m, f \mid Q$ is Lipschitz. By 2.17 and 2.14, $a Q$ is a Lipschitz cone. Hence $f \mid P \cap A$ is Lipschitz.

2.19. Theorem. Let $a Q$ be a cone in $R^{n}$ such that $Q$ is a compact polyhedron. Then $a Q$ is a Lipschitz cone.

Proof. Let $c: Q \rightarrow R^{1}$ be the constant map $c(x)=1$. Since $c$ is PL, its 0-cone $\bar{c}: a Q \rightarrow R^{1}$ is also PL. By 2.18, $\bar{c}$ is Lipschitz. By $2.13(2), a Q$ is a Lipschitz cone.

2.20. Standard mistakes. The well-known standard mistake of PL topology is a radial projection of a compact polyhedron onto another. It is usually not PL. By 2.13 and 2.19, such a map is always Lipschitz. A standard mistake of another kind will be considered in 2.43 .

2.21. Unions. Let $A \cup B$ be a metric space. Let $f: A \cup B \rightarrow Y$ be a function such that $f \mid A$ and $f \mid B$ are LIP. Without any additional condition, $f$ need not be LIP or even continuous. If $A \backslash B$ and $B \backslash A$ are separated, that is, $\overline{A \backslash B} \cap$ $\cap(B \backslash A)=\emptyset=(A \backslash B) \cap \overline{A \backslash B}$, then $f$ is continuous. In this case we say that $A \cup B$ is a proper union of $A$ and $B$. If $A$ and $B$ are both open or both closed in $A \cup B$, then $A \cup B$ is a proper union. In particular, the union of two compact sets is always proper.

Even if $A$ and $B$ are compact, $f$ need not be LIP. For example, let $A$ be the parabolic arc $\left\{(x, y) \in R^{2} \mid 0 \leqq x \leqq 1, y=x^{2}\right\}$, let $B=\{(x, y) \mid(x,-y) \in A\}$, and let $f: A \cup B \rightarrow R^{1}$ be defined by $f(x, y)=x$ for $x \in A$ and by $f(x, y)=-x$ for $(x, y) \in B$. Then $f \mid A$ and $f \mid B$ are Lipschitz, but $f$ is not.

We say that $A \cup B$ is a LIP proper union of $A$ and $B$ if $A \cup B$ is a proper union and if a function $f: A \cup B \rightarrow Y$ is LIP whenever $f \mid A$ and $f \mid B$ are LIP. A related concept has been considered by Wilker [32].

We shall give some characterizations for LIP proper unions. Before that, we give a simple but useful result, which can often be directly used to prove that a map is Lipschitz.

2.22. Lemma. Suppose that $A \cup B$ is a metric space, that $E \subset A \cup B$, and that $C \geqq 1$ is a constant such that for every pair of points $a \in A \cap E, b \in B \cap E$, there 
is $c \in A \cap B$ such that $d(a, c)+d(c, b) \leqq C d(a, b)$. If $f: A \cup B \rightarrow Y$ is a function such that $f \mid A$ and $f \mid B$ are L-Lipschitz, then $f \mid E$ is CL-Lipschitz.

Proof. For $a \in A \cap E, \quad b \in B \cap E, \quad$ we obtain $d^{\prime}(f(a), f(b)) \leqq d^{\prime}(f(a), f(c))+$ $d^{\prime}(f(c), f(b)) \leqq L d(a, c)+L d(c, b) \leqq C L d(a, b)$.

2.23. Theorem. Let $A \cup B$ be a proper union with $A \cap B \neq \emptyset$. Define $u: A \cup B \rightarrow R^{1}$ by $u(x)=d(x, A \cap B)$ for $x \in A$ and by $u(x)=0$ for $x \in B$. Then $A \cup B$ is a LIP proper union if and only if $u$ is LIP.

Proof. The necessity of the condition is clear. Conversely, assume that $u$ is LIP. Let $f: A \cup B \rightarrow Y$ be a map such that $f \mid A$ and $f \mid B$ are LIP, and let $x \in A \cup B$. If $x \in A \backslash B$, there is a neighborhood $U$ of $x$ in $A \cup B$ with $U \subset A$. Then $f \mid U$ is LIP. Next suppose $x \in A \cap B$. Choose $r>0$ such that for $U=B(x, 2 r), f \mid A \cap U$, $f \mid B \cap U$, and $u \mid U$ are Lipschitz. Let $a \in A \cap B(x, r)$ and $b \in B \cap B(x, r), a \neq b$. Pick a point $c \in A \cap B \cap U$ such that $d(a, c)<d(a, A \cap B)+d(a, b)$. Then $d(a, c)+$ $+d(c, b) \leqq 2 d(a, c)+d(a, b) \leqq 2(u(a)-u(b))+3 d(a, b) \leqq(2 \operatorname{lip}(u \mid U)+3) d(a, b) . \quad$ By 2.22, $f \mid B(x, r)$ is Lipschitz.

2.24. Theorem. Let $A \cup B$ be a proper union with $A \cap B \neq \emptyset$. Then $A \cup B$ is a LIP proper union if and only if

$$
\lim _{r \rightarrow 0} \sup \left\{\frac{d(a, A \cap B)}{d(a, B \backslash A)} \mid a \in(A \backslash B) \cap B(x, r)\right\}<\infty
$$

for all $x \in \overline{A \backslash B} \cap \overline{B \backslash A} \subset A \cap B$.

Proof. Suppose that $A \cup B$ is a LIP proper union. Let $x \in \overline{A \backslash B} \cap \bar{B} \backslash A$. By 2.23, there are $r>0$ and $L \geqq 1$ such that for $U=B(x, 2 r), u \mid U$ is $L$-Lipschitz. Let $a \in(A \backslash B) \cap B(x, r)$ and $b \in(B \backslash A) \cap U$. Then $d(a, A \cap B)=|u(a)-u(b)| \leqq$ $\leqq L d(a, b)$. Hence $d(a, A \cap B) \leqq L d(a, B \backslash A)$, and the limit is finite.

Conversely, assume that the limit is finite for all $x$. Let $x \in \overline{A \backslash B} \cap \overline{B \backslash A}$. There is a neighborhood $U$ of $x$ and a constant $L \geqq 1$ such that $d(a, A \cap B) \leqq L d(a, B \backslash A)$ for all $a \in(A \backslash B) \cap U$. Then $|u(a)-u(b)| \leqq L d(a, b)$ for all $a \in U \cap A$ and $b \in B \cap U$. Thus $u \mid U$ is Lipschitz, and the theorem follows from 2.23 .

2.25. We next turn to the case where $A$ and $B$ are subsets of $R^{n}$ with $A \cap B \neq \emptyset$. For $a \in A, b \in B$, we set $\alpha(A, B, a, b)=\sup \{\operatorname{ang}(a-y, b-y) \mid y \in A \cap B\}$. The intersection angle of $A$ and $B$ at a point $x \in A \cap B$ is defined by

$$
\alpha(A, B, x)=\liminf _{a, b \rightarrow x} \alpha(A, B, a, b) .
$$

For example, if $A$ and $B$ are line segments $a x$ and $b x$ with $x=A \cap B$, then $\alpha(A, B, x)$ is the ordinary angle between $A$ and $B$. Note that $\alpha(A, B, 0)=0$ for the example in 2.21 .

2.26. Theorem. Let $A \cup B$ be a proper union of $A, B \subset R^{n}$. If $\alpha(A, B, x)>0$ for all $x \in A \cap B$, then $A \cup B$ is a LIP proper union. 
Proof. We may assume $A \cap B \neq \emptyset$. Let $u: A \cup B \rightarrow R^{1}$ be the map given in 2.23, and let $x \in A \cap B$. It suffices to show that $u$ is Lipschitz in a neighborhood of $x$. Set $\varepsilon=\alpha(A, B, x) / 2$ and choose a neighborhood $U$ of $x$ such that $\alpha(A, B, a, b)>\varepsilon$ for all $a \in(A \backslash B) \cap U$ and $b \in(B \backslash A) \cap U$. For such a pair $a, b$ choose $y \in A \cap B$ such that ang $(a-y, b-y)>\varepsilon$. Then $|u(a)-u(b)| /|a-b| \leqq|a-y| /|a-b| \leqq 1 / \sin \varepsilon$.

2.27. Remark. The converse of 2.26 is not true. A counterexample is given in 3.10 (4).

2.28. Remark. Sometimes the LIP properness of a union $A \cup B$ depends only on the space $A \cup B$ and not on the sets $A, B$. For example, let $A \cup B$ be a proper union such that $A \cup B$ is a convex subset of $R^{n}$. Then $\alpha(A, B, x)=\pi$ for all $x \in A \cap B$, and the LIP properness follows from 2.26 .

We shall next consider this phenomenon in a more general situation.

2.29. Quasiconvexity. Let $X$ be a metric space. Given a pair of points $a, b \in X$, we let $C(a, b, X)$ denote the infimum of all numbers $C \geqq 1$ such that there is a rectifiable path $\gamma$ in $X$ joining $a$ and $b$ with length $l(\gamma) \leqq C d(a, b)$. If no such path exists, we set $C(a, b, X)=\infty$. For $A \subset X$ we write $C(A, X)=\sup \{C(a, b, X) \mid a \in A, b \in A\}$ and $C(X)=C(X, X)$. If $C(X)<\infty$, we say that $X$ is quasiconvex, and $X$ is $C$-quasiconvex if $C(X) \leqq C$. If each point of $X$ has a neighborhood $U$ such that $C(U, X)<\infty$, $X$ is locally quasiconvex.

For example, a convex set in a normed vector space is 1-quasiconvex. The spheres $S^{n}$ are $(\pi / 2)$-quasiconvex $(n \geqq 1)$. A quasiconvex space is always locally quasiconvex. The arc $\left\{(x, y) \in R^{2}|0 \leqq x \leqq 1| y \mid,=x^{2}\right\}$ is not locally quasiconvex.

2.30. Lemma. Every open subset of a locally quasiconvex metric space is locally quasiconvex.

Proof. Let $V$ be open in a locally quasiconvex space $X$. For $x \in V$ choose a neighborhood $U$ such that $C(U, X)=C<\infty$. Next choose $r>0$ such that $B(x,(2 C+3) r) \subset U \cap V$. Let $W$ be the ball $B(x, r)$. If $a, b \in W$, there is a path $\gamma$ joining $a$ and $b$ in $X$ such that $l(\gamma) \leqq(C+1) d(a, b)$. If $y$ is any point on im $\gamma$, then $d(a, y) \leqq l(\gamma) \leqq 2(C+1) r$. Hence im $\gamma \subset V$, which implies $C(W, V) \leqq C+1$.

2.31. Retracts. A subset $A$ of a metric space $X$ is a Lipschitz retract of $X$ if there is a Lipschitz map $r: X \rightarrow A$ with $r \mid A=\mathrm{id}$. If $A$ is a Lipschitz retract of a neighborhood of $A$, then $A$ is a Lipschitz neighborhood retract of $X$. Similarly, we define the concepts LIP retract and LIP neighborhood retract.

2.32. Lemma. A Lipschitz retract of a quasiconvex space is quasiconvex. A LIP neighborhood retract of a locally quasiconvex space is locally quasiconvex.

Proof. Suppose that $X$ is $C$-quasiconvex and that $r: X \rightarrow A$ is an $L$-Lipschitz retraction. Given $a, b \in A$ and $\varepsilon>0$, there is a path $\gamma$ joining $a$ and $b$ in $X$ with 
$l(\gamma) \leqq(C+\varepsilon) d(a, b)$. Then $r \gamma$ joins $a$ and $b$ in $A$, and $l(r \gamma) \leqq L(C+\varepsilon) d(a, b)$. Thus $A$ is $L C$-quasiconvex.

Next assume that $r: V \rightarrow A$ is a LIP retraction of a neighborhood $V$ of $A$. Let $x \in A$ and choose an open neighborhood $U$ of $x$ in $X$ such that $U \subset V$ and $\operatorname{lip}(r \mid U)=L<\infty$. By 2.30, there is a neighborhood $W \subset U$ of $x$ with $C(W, U)=$ $C<\infty$. Arguing as in the first part of the proof we see that $C(A \cap W, A) \leqq L C$. Hence $A$ is locally quasiconvex.

2.33. Lemma. If $X$ is compact, connected, and locally quasiconvex, then $X$ is quasiconvex.

Proof. Since $X$ is connected and locally quasiconvex, each pair of points $x, y \in X$ can be joined by a rectifiable path in $X$. Let $\delta(x, y)$ be the infimum of the lengths of all such paths. Then $\delta$ is a metric in $X$. Since $X$ is locally quasiconvex, the identity map id: $(X, d) \rightarrow(X, \delta)$ is LIP. Since $X$ is compact, it is Lipschitz, whence $X$ is quasiconvex.

2.34. Theorem. Every compact connected polyhedron in $R^{n}$ is quasiconvex. Every polyhedron in $R^{n}$ is locally quasiconvex.

Proof. It suffices to prove the first assertion. Let $P \subset R^{n}$ be a compact connected polyhedron. Then $P$ is a PL retract of a regular neighborhood $N$ of $P$. By 2.18, $P$ is a Lipschitz neighborhood retract of $R^{n}$. The theorem follows from 2.32 and 2.33 .

2.35. Theorem. Suppose that $X$ is $C$-quasiconvex and that $\mathscr{A}$ is a cover of $X$ such that for each $x \in X$, the star st $(x, \mathscr{A})=\cup\{A \mid x \in A \in \mathscr{A}\}$ of $x$ is a neighborhood of $x$. (For example, $\mathscr{A}$ is a locally finite closed cover of $X$ or $\mathscr{A}$ is a family of two sets with a proper union.) If $f: X \rightarrow Y$ is a function such that $f \mid A$ is L-Lipschitz for all $A \in \mathscr{A}$, then $f$ is $C L$-Lipschitz.

Proof. Let $a, b \in X$, and let $\varepsilon>0$. Choose a path $\gamma: I \rightarrow X$ with $\gamma(0)=a$, $\gamma(1)=b, l(\gamma) \leqq(C+\varepsilon) d(a, b)$. For every $s \in I$, choose an open interval neighborhood $U(s)$ in $I$ such that $\gamma U(s) \subset \operatorname{st}(\gamma(s), \mathscr{A})$. Choose a subcover $\left\{U\left(s_{i}\right) \mid 1 \leqq i \leqq k\right\}$ of $\{U(s) \mid s \in I\}$ which has no proper subcover. We may assume that $s_{i}<s_{i+1}$ and $\emptyset \neq U\left(s_{i}\right) \cap U\left(s_{i+1}\right) \subset\left(s_{i}, s_{i+1}\right)$ for all $i \in\{1, \ldots, k-1\}$. Choosing numbers $s_{i}^{\prime} \in U\left(s_{i}\right) \cap$ $\cap U\left(s_{i+1}\right)$ and relabeling the sequence $\left(0, s_{1}, s_{1}^{\prime}, \ldots, s_{k-1}^{\prime}, s_{k}, 1\right)$ we obtain numbers $0=t_{0}<\ldots<t_{2 k}=1$ such that for $x_{i}=\gamma\left(t_{i}\right),\left\{x_{i-1}, x_{i}\right\}$ is contained in some member of $\mathscr{A}$. Thus $d^{\prime}\left(f\left(x_{i-1}\right), f\left(x_{i}\right)\right) \leqq L d\left(x_{i-1}, x_{i}\right)$, which implies

$$
d^{\prime}(f(a), f(b)) \leqq L \sum_{i=1}^{2 k} d\left(x_{i-1}, x_{i}\right) \leqq L l(\gamma) \leqq(C+\varepsilon) L d(a, b) .
$$

2.36. Theorem. Suppose that $X$ is locally quasiconvex and that $\mathscr{A}$ is a pointfinite cover of $X$ such that for each $x \in X$, st $(x, \mathscr{A})$ is a neighborhood of $x$. If $f: X \rightarrow Y$ is a function such that $f \mid A$ is LIP for all $A \in \mathscr{A}$, then $f$ is LIP. 
Proof. Let $x \in X$, and let $A_{1}, \ldots, A_{k}$ be the members of $\mathscr{A}$ containing $x$. Choose an open neighborhood $U$ of $x$ and a positive number $L$ such that $U \subset \operatorname{st}(x, \mathscr{A})=A_{1} \cup \ldots \cup A_{k}$ and such that $f \mid U \cap A_{i}$ is $L$-Lipschitz for all $i$. Since $f$ is clearly continuous, it follows that also $f \mid U \cap \bar{A}_{i}$ is $L$-Lipschitz for all $i$. By 2.30, there is a neighborhood $V \subset U$ of $x$ with $C(V, U)=C<\infty$. We show that $f \mid V$ is $C L$-Lipschitz. Let $a, b \in V$ and $\varepsilon>0$. Choose a path $\gamma$ joining $a$ and $b$ in $U$ with $l(\gamma) \leqq(C+\varepsilon) d(a, b)$. We can find numbers $0=t_{0}<\ldots<t_{s}=1$ such that for each $i \in\{1, \ldots, s\},\left\{\gamma\left(t_{i-1}\right), \gamma\left(t_{i}\right)\right\}$ is contained in some of the sets $U \cap \bar{A}_{i}$. Hence $d^{\prime}\left(f\left(\gamma\left(t_{i-1}\right)\right), f\left(\gamma\left(t_{i}\right)\right)\right) \leqq L d\left(\gamma\left(t_{i-1}\right), \gamma\left(t_{i}\right)\right)$, which implies

$$
d^{\prime}(f(a), f(b)) \leqq L \sum_{i=1}^{s} d\left(\gamma\left(t_{i-1}\right), \gamma\left(t_{i}\right)\right) \leqq L l(\gamma) \leqq(C+\varepsilon) L d(a, b) .
$$

2.37. Corollary. If $A \cup B$ is locally quasiconvex and a proper union, then $A \cup B$ is a LIP proper union.

2.38. Theorem. If $A$ and $B$ are polyhedra with a proper union, then $A \cup B$ is a LIP proper union.

Proof. Since a proper union of polyhedra is obviously a polyhedron, the theorem follows from 2.34 and 2.37 .

2.39. Cartesian products. Let $(X, d)$ and $\left(X^{\prime}, d^{\prime}\right)$ be metric spaces. Then the distance between points $\left(x, x^{\prime}\right)$ and $\left(y, y^{\prime}\right)$ in $X \times X^{\prime}$ can be defined in three natural ways: $d(x, y)+d^{\prime}\left(x^{\prime}, y^{\prime}\right), \quad\left(d(x, y)^{2}+d^{\prime}\left(x^{\prime}, y^{\prime}\right)^{2}\right)^{1 / 2}, \quad$ or $\max \left(d(x, y), d^{\prime}\left(x^{\prime}, y^{\prime}\right)\right)$. Letting $d_{1}, d_{2}, d_{3}$ denote the corresponding metrics of $X \times X^{\prime}$, we have $d_{3} \leqq d_{2} \leqq$ $\leqq d_{1} \leqq 2 d_{3}$. Hence these metrics are Lipschitz equivalent. From the point of view of LIP topology, it makes no difference which metric we use.

It is readily seen that cartesian products of LIP maps are again LIP. Moreover, a map $\left(f, f^{\prime}\right)$ of a metric space $Z$ into $X \times X^{\prime}$ is LIP if and only if $f$ and $f^{\prime}$ are LIP.

2.40. Maps of $X \times R^{1}$. Let $X$ be a metric space. We shall later make use of maps $f: X \times R^{1} \rightarrow X \times R^{1}$ of the following type: Let $\alpha_{i}$ and $\beta_{i}$ be LIP maps $X \rightarrow R^{1}$, $i=0, \ldots, k$, such that $\alpha_{0}<\ldots<\alpha_{k}$ and $\beta_{0}<\ldots<\beta_{k}$. Then $f(x, t)=\left(x, r_{x}(t)\right)$ where $r_{x}$ is the PL homeomorphism of $R^{1}$ onto $R^{1}$ which maps $\left[\alpha_{i-1}(x), \alpha_{i}(x)\right]$ affinely onto $\left[\beta_{i-1}(x), \beta_{i}(x)\right]$ and is a translation on $\left(-\infty, \alpha_{0}(x)\right]$ and on $\left[\alpha_{k}(x), \infty\right)$.

We shall show that $f$ is a lipeomorphism of $X \times R^{1}$ onto itself. Since $f^{-1}$ is obtained by changing the roles of $\alpha_{i}$ and $\beta_{i}$, it suffices to show that $f$ is LIP. For this, it is sufficient to prove that the map $r: X \times R^{1} \rightarrow R^{1}$, defined by $r(x, t)=r_{x}(t)$, is LIP.

Set $A_{i}=\left\{(x, t) \mid \alpha_{i-1}(x) \leqq t \leqq \alpha_{i}(x)\right\}$ for $1 \leqq i \leqq k$ and $A_{0}=\left\{(x, t) \mid t \leqq \alpha_{0}(x)\right\}$, $A_{k+1}=\left\{(x, t) \mid t \geqq \alpha_{k}(x)\right\}$. For $(x, t) \in A_{1} \quad$ we have $r(x, t)=(1-u) \beta_{0}(x)+u \beta_{1}(x)$ 
where $u=\left(t-\alpha_{0}(x)\right) /\left(\alpha_{1}(x)-\alpha_{0}(x)\right)$. Using 2.4 we see that $r \mid A_{1}$ is LIP. A similar proof shows that $r \mid A_{i}$ is LIP for all $i$.

To show that $r$ is LIP in $X \times R^{1}$ we use 2.22. For example, let us show that $r \mid A_{1} \cup A_{2}$ is LIP. Since the question is local, we may assume that $\operatorname{lip} \alpha_{1}=L<\infty$. Let $a=(x, s) \in A_{1}$ and $b=(y, t) \in A_{2}$. Then $c=\left(x, \alpha_{1}(x)\right) \in A_{1} \cap A_{2}$. Using the metric $d_{1}$ of 2.39 in $X \times R^{1}$ we have $d_{1}(a, c)=\alpha_{1}(x)-s$ and $d_{1}(c, b)=d(x, y)+$ $+\left|t-\alpha_{1}(x)\right|$. If $t \geqq \alpha_{1}(x)$, we obtain $d_{1}(a, c)+d_{1}(c, b)=d_{1}(a, b)$. If $t<\alpha_{1}(x)$, then $t \geqq \alpha_{1}(y) \geqq \alpha_{1}(x)-L d(x, y)$, which implies $d_{1}(a, c)+d_{1}(c, b) \leqq t-s+(2 L+1) d(x, y) \leqq$ $\leqq(2 L+1) d_{1}(a, b)$. Hence $r \mid A_{1} \cup A_{2}$ is LIP.

2.41. Lemma. Let $C$ be $a(p+q+1)$-simplex which is the join of a p-simplex $A$ and $a$-simplex $B, p \geqq 0, q \geqq 0$. Let $\pi: A \times B \times I \rightarrow C$ be the map $\pi(x, y, t)=$ $=(1-t) x+t y$. Then $\pi$ is LIP and defines a lipeomorphism $\pi_{0}: A \times B \times(0,1) \rightarrow$ $\rightarrow C \backslash(A \cup B)$.

Proof. Using an auxiliary affine homeomorphism, we may assume that $C \subset R^{p+q+1}=R^{p} \times R^{q} \times R^{1}, A \subset R^{p} \times 0 \times 0, B \subset 0 \times R^{q} \times 1$. Choose $R>0$ such that $C \subset B^{p+q+1}(R)$. Let $z_{1}=\left(x_{1}, y_{1}, t_{1}\right)$ and $z_{2}=\left(x_{2}, y_{2}, t_{2}\right) \in A \times B \times I$. Then

Hence $\pi$ is LIP.

$$
\begin{aligned}
\left|\pi\left(z_{1}\right)-\pi\left(z_{2}\right)\right| & \leqq\left(1-t_{1}\right)\left|x_{1}-x_{2}\right|+\left|t_{1}-t_{2}\right|\left|x_{2}\right|+t_{1}\left|y_{1}-y_{2}\right|+\left|t_{1}-t_{2}\right|\left|y_{2}\right| \\
& \leqq(2+2 R)\left|z_{1}-z_{2}\right| .
\end{aligned}
$$

Let $0<\delta<1 / 2$, and let $z_{1}, z_{2} \in A \times B \times(\delta, 1-\delta)$. Then $\delta\left|x_{1}-x_{2}\right| \leqq \mid\left(1-t_{1}\right) x_{1}-$ $-\left(1-t_{2}\right) x_{2}|+| t_{2}-t_{1}|| x_{2}|\leqq(1+R)| \pi\left(z_{1}\right)-\pi\left(z_{2}\right) \mid, \quad$ and $\quad$ similarly $\quad \delta\left|y_{1}-y_{2}\right| \leqq(1+$ $R)\left|\pi\left(z_{1}\right)-\pi\left(z_{2}\right)\right|$, which implies $\left|z_{1}-z_{2}\right| \leqq(1+(2+2 R) / \delta)\left|\pi\left(z_{1}\right)-\pi\left(z_{2}\right)\right|$. Hence $\pi_{0}$ is a lipeomorphism.

2.42. Stretching maps. We shall consider the stretching process between dual skeletons, which is often used in an engulfing argument. Suppose that $K$ is a finite simplicial complex and that $L, M$ are disjoint subcomplexes of $K$ such that every simplex of $K$ is the join of a simplex in $L$ and a simplex in $M$. Let $X$ be the set of all $(x, y) \in|L| \times|M|$ such that $x y$ lies in a simplex of $K$, and let $\alpha, \beta: X \rightarrow(0,1)$ be LIP maps. Choose $t_{1}, t_{2} \in(0,1)$ such that $\operatorname{im} \alpha \cup \operatorname{im} \beta \subset\left(t_{1}, t_{2}\right)$. For each $(x, y) \in X$ we let $\omega_{x y}: I \rightarrow I$ denote the PL homeomorphism which maps the points $0, t_{1}$, $\alpha(x, y), t_{2}, 1$ to the points $0, t_{1}, \beta(x, y), t_{2}, 1$, and is affine on the remaining intervals. Let $h:|K| \rightarrow|K|$ be the unique bijective function such that $h|| L|\cup| M \mid=$ id and $h((1-t) x+t y)=\left(1-\omega_{x y}(t)\right) x+\omega_{x y}(t) y$ for all $(x, y) \in X$. We shall prove that $h:|K| \rightarrow|K|$ is a lipeomorphism.

Since $h^{-1}$ is obtained from $h$ by changing the roles of $\alpha$ and $\beta$, it suffices to show that $h$ is LIP. By 2.38, it suffices to show that $h \mid C$ is LIP for every $C \in K$. Write $C=A B$ with $A \in L, B \in M$. We may assume that $A \neq \emptyset \neq B$. Let $\pi: A \times B \times I \rightarrow C$ be the map of 2.41. Since $C$ is convex, it suffices to show that $h$ is LIP in each of the sets $C_{i}=\pi D_{i}, \quad i=1,2$, where $D_{1}=A \times B \times\left(\left[0, t_{1}\right] \cup\left[t_{2}, 1\right]\right)$ and $D_{2}=A \times B \times$ 
$\times\left[t_{1}, t_{2}\right]$. Since $h \mid C_{1}=\mathrm{id}$, it is LIP. Furthermore, we may write $h\left|C_{2}=\pi_{0} g \pi_{0}^{-1}\right| C_{2}$ where $\pi_{0}$ is the lipeomorphism of 2.41 , and $g(x, y, t)=\left(x, y, \omega_{x y}(t)\right)$. It follows from 2.40 that $g$ is LIP. Hence $h$ is LIP.

The stretching process will be used in 8.4 in the following situation. $K$ has a subcomplex $K_{1}$ such that $|K|$ and $\left|K_{1}\right|$ are concentric $n$-cubes, $L$ is the 2-skeleton of $K$, and $M$ is the dual $(n-3)$-skeleton of a derived subdivision of $K$. Given open neighborhoods $U$ and $V$ of $|L|$ and $|M|$, we need a lipeomorphism $h:|K| \rightarrow|K|$ such that $\left|K_{1}\right| \subset h U \cup V$ and $h|\partial| K \mid=\mathrm{id}$. For this, we first choose $\varepsilon \in(0,1 / 2)$ such that for all $(x, y, t) \in X \times[0, \varepsilon],(1-t) x+t y \in U$ and $t x+(1-t) y \in V$. Then choose a LIP map $\lambda:|K| \rightarrow[\varepsilon, 1 / 2]$ such that $\lambda(x)=\varepsilon$ for $x \in\left|K_{1}\right|$ and $\lambda(x)=1 / 2$ for $x \in \partial|K|$, and set $\alpha(x, y)=(\lambda(x)+\lambda(y)) / 2, \beta(x, y)=1-\alpha(x, y)$. Then the stretching map $h:|K| \rightarrow|K|$ has the desired properties.

2.43. Remark. Suppose that the maps $\alpha$ and $\beta$ of 2.42 are constants, $\alpha \neq \beta$. It is sometimes stated that the corresponding homeomorphism $h:|K| \rightarrow|K|$ is PL. However, this is usually not true, for example if $|K|$ is a triangle $a b c,|L|=a$, $|M|=b c$. We may call this map a standard mistake of the second kind. A PL homeomorphism is easily obtained as follows: Let $f: K \rightarrow I$ be the simplicial map which maps $L$ into 0 and $M$ into 1 . Choose deriveds $K_{1}, K_{2}$ of $K$ near $L$ [25, p. 32] such that $f^{-1}[0, \alpha]$ and $f^{-1}[0, \beta]$ are the underlying polyhedra of the derived neighborhoods $N\left(L, K_{1}\right)$ and $N\left(L, K_{2}\right)$, respectively. Then the canonical simplicial isomorphism $\varphi: K_{1} \rightarrow K_{2}$ maps $N\left(L, K_{1}\right)$ onto $N\left(L, K_{2}\right)$.

\section{Lipschitz manifolds}

3.1. Definition. A Lipschitz n-manifold (or a LIP $n$-manifold) is a separable metric space $M$ such that every point $x \in M$ has a closed neighborhood $U$ lipeomorphic to $I^{n}$.

3.2. Remarks. (1) Recall that a connected paracompact topological manifold has a countable base. Hence, separability is not a restriction for connected manifolds.

(2) Since $I^{n}$ is lipeomorphic to $\bar{B}^{n}$, the pair $(U, x)$ in 3.1 is lipeomorphic to either $\left(I^{n}, 0\right)$ or $\left(I^{n}, e^{n}\right)$. It follows that each point of a LIP manifold has an open neighborhood lipeomorphic to either $R^{n}$ or $R_{+}^{n}$.

(3) The boundary $\partial M$ of a LIP $n$-manifold is either empty or a LIP $(n-1)$ manifold.

(4) If $M$ and $N$ are LIP manifolds, so is $M \times N$.

(5) A LIP manifold is locally quasiconvex.

3.3. Atlases. There is an alternative way to define a LIP manifold based on atlases. Let $M$ be a Hausdorff space. A LIP atlas on $M$ is a family of charts $\left(U_{i}, h_{i}\right)$ where the sets $U_{i}$ form an open cover of $M, h_{i}$ maps $U_{i}$ homeomorphically onto a 
set $U_{i}^{\prime}$ which is open either in $R^{n}$ or in $R_{+}^{n}$, and $h_{i} h_{j}^{-1}$ defines a lipeomorphism of $h_{j}\left[U_{i} \cap U_{j}\right]$ onto $h_{i}\left[U_{i} \cap U_{j}\right]$ for all $i$ and $j$. Two LIP atlases are called equivalent if their union is a LIP atlas. Then a LIP manifold can be defined as a pair consisting of $M$ and an equivalence class of LIP atlases. The concept of a LIP map between LIP manifolds is then defined in the obvious way using charts.

If $M$ is a LIP manifold in the sense of 3.1, it has a natural LIP atlas consisting of all lipeomorphisms $h: U \rightarrow U^{\prime}$ such that $U$ is open in $M$ and $U^{\prime}$ is open in $R^{n}$ or in $R_{+}^{n}$. Moreover, the two definitions of LIP maps of $M$ and into $M$ are consistent. One can show that a paracompact LIP manifold is lipeomorphic to a metric space. Hence, for second countable spaces, the atlas definition is not essentially more general than 3.1. This can be proved in several ways. For example, it will follow from our embedding theorem 4.2. But it is also a special case of a metrization theorem for locally metric spaces (Theorem 3.5 and Remark 3.7), which is our next goal. Moreover, Weller [30] has proved the result (stated for closed manifolds) using a method somewhat similar to the proof of 3.5 .

3.4. Local metrics. A local metric on a Hausdorff space $X$ is a family of metric spaces $\left(U_{i}, d_{i}\right)$ such that the sets $U_{i}$ form an open cover of $X, d_{i}$ is compatible with the topology of $U_{i}$, and for each pair $i, j$ of indices, the restrictions of $d_{i}$ and $d_{j}$ are LIP equivalent on $U_{i} \cap U_{j}$. Two local metrics on $X$ are called LIP equivalent if their union is a local metric. Cf. Whitehead [31, p. 166]. A locally metric space is a pair consisting of a Hausdorff space $X$ and an equivalence class of local metrics on $X$. Each metric space defines a locally metric space in the obvious way. We shall show that each paracompact locally metric space can be obtained in this way.

Every LIP atlas $\left(U_{i}, h_{i}\right)_{i \in I}$ of a manifold $M$ defines a local metric on $M$ consisting of pairs $\left(U_{i}, d_{i}\right)$ where $d_{i}(x, y)=\left|h_{i}(x)-h_{i}(y)\right|$. Moreover, equivalent LIP atlases define LIP equivalent local metrics. Hence every LIP manifold in the atlas sense can be regarded as a locally metric space. The same is true for abstract PL and DIFF manifolds.

3.5. The orem (Metrization). On a paracompact Hausdorff space, every local metric is LIP equivalent to a metric.

Proof. Suppose that $\delta=\left(U_{i}, d_{i}\right)_{i \in I}$ is a local metric on a paracompact Hausdorff space $X$. We may assume that the cover $\left(U_{i}\right)_{i \in I}$ is locally finite. We first show that $\delta$ is LIP equivalent to a local metric $\delta^{\prime}=\left(U_{j}^{\prime}, d_{j}^{\prime}\right)_{j \in J}$ of $X$ such that for every pair $j, k \in J, d_{j}^{\prime}$ and $d_{k}^{\prime}$ are Lipschitz (not only LIP) equivalent in $U_{j}^{\prime} \cap U_{k}^{\prime}$. There is an open cover $\left(V_{i}\right)_{i \in I}$ of $X$ with $\bar{V}_{i} \subset U_{i}$ for all $i$. For every $x \in X$, choose an open neighborhood $W(x)$ such that (1) $W(x) \subset V_{i}$ if $x \in V_{i}$, (2) $W(x) \subset U_{i}$ if $x \in U_{i}$, (3) $W(x) \cap \bar{V}_{i}=\emptyset$ if $x \notin \bar{V}_{i}$, (4) $d_{i}$ and $d_{j}$ are Lipschitz equivalent in $W(x)$ if $x \in U_{i} \cap U_{j}$. Next choose a locally finite open refinement $\left(U_{j}^{\prime}\right)_{j \in J}$ of the cover $(W(x))_{x \in X}$. For every $j \in J$, choose $x_{j} \in X$ and $i(j) \in I$ such that $U_{j}^{\prime} \subset W\left(x_{j}\right) \subset V_{i(j)}$. Then $d_{j}^{\prime}=$ $=d_{i(j)} \mid U_{j}^{\prime} \times U_{j}^{\prime}$ is a metric for $U_{j}^{\prime}$, compatible with the topology of $U_{j}^{\prime}$. Suppose 
that $U_{j}^{\prime}$ meets $U_{k}^{\prime}$. Then $W\left(x_{k}\right)$ meets $V_{i(j)}$, which implies $x_{k} \in \bar{V}_{i(j)}$. Thus $x_{k} \in U_{i(j)} \cap$ $\cap U_{i(k)}$, whence $d_{j}^{\prime}$ and $d_{k}^{\prime}$ are Lipschitz equivalent in $W\left(x_{k}\right)$, and hence in $U_{j}^{\prime} \cap U_{k}^{\prime}$. Thus the local metric $\delta^{\prime}=\left(U_{j}^{\prime}, d_{j}^{\prime}\right)_{j \in J}$ has the desired property. Obviously $\delta$ and $\delta^{\prime}$ are LIP equivalent.

Changing notation, we assume that the original local metric $\delta=\left(U_{i}, d_{i}\right)_{i \in I}$ has the above property. Choose again an open cover $\left(V_{i}\right)_{i \in I}$ of $X$ with $\bar{V}_{i} \subset U_{i}$. We define a function $d: X \times X \rightarrow R^{1}$ as follows: For $x, y \in X$, let $P(x, y)$ be the set of all finite sequences of the form $\pi=\left(x_{0}, \ldots, x_{k} ; i_{1}, \ldots, i_{k}\right)$ such that $x_{0}=x, x_{k}=y$, and $\left\{x_{j-1}, x_{j}\right\} \subset V_{i_{j}}$. For such $\pi$, set

$$
s(\pi)=\sum_{j=1}^{k} d_{i_{j}}\left(x_{j-1}, x_{j}\right) .
$$

If $P(x, y)=\emptyset$, we set $d(x, y)=1$, otherwise

$$
d(x, y)=\min (1, \inf \{s(\pi) \mid \pi \in P(x, y)\}) .
$$

It is easy to see that $d$ is a pseudometric for the set $X$.

For the rest of the proof we fix the following notation: Let $x \in X$ and choose $i \in I$ such that $x \in V_{i}$. Choose an open neighborhood $V \subset V_{i}$ of $x$ such that $J_{1}=$ $=\left\{j \in I \mid V\right.$ meets $\left.U_{j}\right\}$ is finite and for every $j \in J_{1}$, either $V \subset U_{j}$ or $V \cap V_{j}=\emptyset$. Set $J_{2}=\left\{j \in J_{1} \mid V \subset U_{j}\right\}$. There is $b>1$ such that $b^{-1} d_{k}(u, v) \leqq d_{j}(u, v) \leqq b d_{k}(u, v)$ for all $j, k$ in $J_{1}$ and for all $u, v$ in $U_{j} \cap U_{k}$. Choose $r \in(0,1)$ such that the ball $B_{d_{j}}(x, r)=$ $=\left\{y \in U_{j} \mid d_{j}(x, y)<r\right\}$ is contained in $V$ for all $j \in J_{2}$.

We must show that $d$ is compatible with the topology of $X$ (hence a metric) and LIP equivalent to $\delta$. If $y, z \in V_{j}$, then $d(y, z) \leqq s(y, z ; j)=d_{j}(y, z)$. Hence id: $X \rightarrow(X, d)$ is continuous, and id: $\left(U_{i}, d_{i}\right) \rightarrow\left(U_{i}, d\right)$ is LIP. We next show that id: $(X, d) \rightarrow X$ is continuous. Let $W$ be a neighborhocd of $x$ in $X$. Replacing $V$ by $V \cap W$, we may assume $V \subset W$. We claim that $d(y, x)<r / b$ implies $y \in W$. Since $r / b<1$, there is $\pi=\left(x_{0}, \ldots, x_{k} ; i_{1}, \ldots, i_{k}\right) \in P(x, y)$ such that $s(\pi)<r / b$. Then $x_{0}=x \in V$. Suppose inductively that $x_{i} \in V$ for all $i \leqq q$. Then $i_{q+1} \in J_{2}$ and $x_{i} \in U_{i_{q+1}}$ for all $i \leqq i_{q+1}$. Hence $d_{i_{q+1}}\left(x_{q+1}, x\right) \leqq b s(\pi)<r$, and thus $x_{q+1} \in V$, which implies $y=x_{k} \in V \subset W$.

It remains to show that $\mathrm{id}:\left(U_{j}, d\right) \rightarrow\left(U_{j}, d_{j}\right)$ is LIP for every $j \in I$. It suffices to show that this map is Lipschitz in a neighborhood of $x$ for every $j \in I$ such that $x \in U_{j}$. Since $\delta$ is a local metric, we may assume $j=i$. The set $B=\cap\left\{B_{d_{j}}(x, r / 2) \mid j \in J_{2}\right\}$ is a neighborhood of $x$ in $U_{i}$. We shall show that $d_{i}(y, z) \leqq 2 b d(y, z)$ for $y, z \in B$. Since $(y, z ; i) \in P(y, z), d(y, z)<1$. Let $\pi=\left(y_{0}, \ldots, y_{k} ; i_{1}, \ldots, i_{k}\right) \in P(y, z)$. If $y_{v} \in V$ for all $v$, then

$$
d_{i}(y, z) \leqq \sum_{v=1}^{k} d_{i}\left(y_{v-1}, y_{v}\right) \leqq b s(\pi) .
$$

If $y_{v} \notin V$ for some $v$, let $q$ be the least $v$ with this property. Then $i_{q} \in J_{2}$, whence $d_{i_{q}}\left(y_{q}, x\right) \geqq r$. Thus $d_{i_{q}}\left(y_{q}, y\right)>r / 2$, which implies $d_{i}(y, z)<r<2 d_{i_{q}}\left(y_{q}, y\right) \leqq 2 b s(\pi)$. Together with (3.6), this implies $d_{i}(y, z) \leqq 2 b d(y, z)$. 
3.7. Remark. Suppose that $M$ is a second countable Hausdorff space with a LIP atlas $\mathscr{A}$. Then it has a natural local metric as in 3.4. Since $M$ is paracompact, it follows from 3.5 that this local metric is LIP equivalent to a metric $d$ on $X$. If $\mathscr{A}$ is replaced by an equivalent LIP atlas $\mathscr{A}^{\prime}$, we obtain a metric $d^{\prime}$ which is LIP equivalent to $d$. To get a full equivalence with the atlas definition, we should define a LIP manifold as a pair consisting of a space $X$ and a LIP equivalence class of metrics of $X$ such that for some (and hence for each) $d$ in the class, $(X, d)$ is locally lipeomorphic to $I^{n}$. However, we prefer to use the conceptually simpler Definition 3.1.

3.8. Terminology. A LIP $n$-ball is a metric space lipeomorphic to $\bar{B}^{n}$. A LIP $n$-sphere is a metric space lipeomorphic to $S^{n}$. A subset $N$ of a LIP manifold $M$ is a LIP submanifold of $M$ if it is a LIP manifold in the metric inherited from $M$. If $N$ is a LIP $q$-submanifold of a LIP $n$-manifold $M$ with $N \subset$ int $M$, we say that $N$ is locally LIP flat at a point $x \in \operatorname{int} N$ if $x$ has a neighborhood $U$ in $M$ such that $(U, U \cap N)$ is lipeomorphic to $\left(V, V \cap R^{q}\right)$ for some $V$ open in $R^{n}$. At a point $x \in \partial N,(U, U \cap N)$ should be lipeomorphic to $\left(V, V \cap R_{+}^{q}\right)$. One can obviously choose $V=R^{n}$.

3.9. Theorem. (Cf. [16]) Let $A$ be a compact convex set in $R^{n}$. Then $A$ is a locally LIP flat LIP ball. In fact, there is a strong lipeomorphism of $R^{n}$ which maps $A$ onto $\bar{B}^{q}, q=\operatorname{dim} A$.

Proof. Let $T$ be the affine subspace of $R^{n}$ spanned by $A$. Choose an interior point $v \in A$ in the topology of $T$. By an auxiliary isometry, we may assume $T=R^{q}$ and $v=0$. Then $A$ is the cone $0(\partial A)$. By 2.17, this cone is a Lipschitz cone, and hence $p(x)=x /|x|$ defines a lipeomorphism $p: \partial A \rightarrow S^{q-1}$. By 2.16, the extended cone $p^{*}: R^{q} \rightarrow R^{q}$ of $p$ is a strong lipeomorphism, and so is $f=p^{*} \times \mathrm{id}: R^{n} \rightarrow R^{n}$. Since $f A=\bar{B}^{q}$, the theorem is proved.

3.10. Examples. (1) By a LIP arc we mean a LIP 1-ball. Unlike PL and DIFF arcs, a LIP arc in $R^{3}$ need not be locally LIP flat. For example, the construction of the Fox-Artin arc, given in [26, pp. 61-62], can be modified so as to yield a LIP arc, which is not even locally TOP flat at the end points. We do not know whether a locally TOP flat LIP arc in $R^{n}$ is always locally LIP flat.

(2) A LIP arc is always rectifiable. Indeed, if $f$ is an $L$-Lipschitz map of $I$ onto $A$, then the length of $A$ is at most $L$. It is not difficult to show that a metric space which is a TOP arc is a LIP arc if and only if it is quasiconvex. For example, the arc $\left\{(x, y) \in R^{2}|0 \leqq x \leqq 1| y \mid,=x^{2}\right\}$ is not a LIP arc. See also Katětov [15, 3.9].

(3) As a rectifiable arc, a LIP arc $A \subset R^{n}$ has a tangent at almost every point. Hence it pierces an $(n-1)$-disk at almost every point. On the other hand, there is a TOP arc in $R^{3}$ which pierces no disk (Bing [2]). This is so wild that no homeomorphism of $R^{3}$ maps it onto a LIP arc.

(4) Although a LIP arc $A \subset R^{n}$ has a tangent at almost every point, its tangential behavior may be fairly complicated at certain points. Let $f: R^{2} \rightarrow R^{2}$ be 
the homeomorphism defined by $f(r, \varphi)=(r, \varphi+\log r)$ in polar coordinates. A computation shows that the derivative of $f$ is bounded in $R^{2} \backslash 0$. Hence $f$ is Lipschitz. Since $f^{-1}(r, \varphi)=(r, \varphi-\log r)$, it similarly follows that $f^{-1}$ is Lipschitz. Thus the image $C$ of the segment joining $(-1,0)$ and $(1,0)$ is a locally LIP flat LIP arc, which consists of two logarithmic spirals and the origin. The origin divides $C$ into two closed LIP subarcs $A, B$. The union $C=A \cup B$ is LIP proper, although the intersection angle $\alpha(A, B, 0)$ is zero. Hence the converse of 2.26 is not true. A similar example in higher dimensions will be given in 4.11 .

(5) Fattening the Fox-Artin arc we obtain a LIP 3-ball $B$ in $R^{3}$ which is not even locally TOP flat. See Gehring [12, Theorem 3, p. 316]. Thus the LIP version of Newman's theorem [25, 3.13] is false. The boundary of $B$ is a LIP 2-sphere in $R^{3}$ which is not locally TOP flat.

(6) The set $\left\{(x, y, z) \in R^{3} \mid 0 \leqq z \leqq 1, x^{2}+y^{2} \leqq z^{4}\right\}$ is a locally TOP flat 3 -ball in $R^{3}$, but it is not locally LIP flat, because it contains no LIP arc through the origin.

3.11. Theorem. Every connected LIP 1-manifold $M$ is lipeomorphic to exactly one of the following LIP 1-manifolds: $(0,1),[0,1),[0,1], S^{1}$.

Proof. It is well known that there is a homeomorphism $f: M_{0} \rightarrow M$ where $M_{0}$ is one of the manifolds listed in the theorem. Using a locally finite cover of $M$ by LIP arcs such that no three of them intersect, we may choose a locally finite family of points $\left(x_{j}\right)_{j \in J}$ in $M_{0}$ with the following properties: (1) The indexing set $J$ is $\mathbb{Z}$ for $M_{0}=(0,1), \mathbf{N}$ for $M_{0}=[0,1)$, and $\{1, \ldots, r\}$ for a compact $M_{0}$. (2) The points $x_{j}$ are in the positive order. (3) If $A_{j}$ is the arc from $x_{j}$ to $x_{j+1}\left(A_{r}\right.$ is from $x_{r}$ to $x_{1}$ if $M_{0}=S^{1}$ ), then $f A_{j}=B_{j}$ is a LIP arc in $M$. (4) The arcs $B_{j}$ cover $M$. Choose a homeomorphism $g: M_{0} \rightarrow M$ such that $g\left(x_{j}\right)=f\left(x_{j}\right)$ for all $j \in J$, and $g$ maps $A_{j}$ lipeomorphically onto $B_{j}$. Since $M_{0}$ and $M$ are locally quasiconvex, it follows from 2.36 that $g$ is a lipeomorphism.

3.12. Corollary. Let $M$ and $N$ be LIP 1-manifolds. If $M$ and $N$ are homeomorphic, they are lipeomorphic.

3.13. Theorem. Let $M$ be a LIP manifold. Then there is a LIP manifold DM, called the double of $M$, which has the following properties: DM contains LIP submanifolds $M_{1}, M_{2}$ such that $D M=M_{1} \cup M_{2}, M_{1} \cap M_{2}=\partial M_{1}=\partial M_{2}$, and there are lipeomorphisms $f_{i}: M \rightarrow M_{i}$ such that $f_{1}\left|\partial M=f_{2}\right| \partial M$. The triple $\left(D M, M_{1}, M_{2}\right)$ is unique up to a lipeomorphism. Moreover, $\partial D M=\emptyset$, and the submanifolds $M_{1}, M_{2}, \partial M_{1}$ are locally LIP flat in DM.

Proof. The uniqueness of $\left(D M, M_{1}, M_{2}\right)$ is clear. If $\partial M=\emptyset$, we may put $D M=M \times\{0,1\}$. If $\partial M \neq \emptyset, D M$ can be constructed for example as follows: Define $f_{1}, f_{2}: M \rightarrow M \times R^{1}$ by $f_{1}(x)=(x, 0)$ and $f_{2}(x)=(x, d(x, \partial M))$. Then each $f_{i}$ is a LIP embedding of $M$ onto $M_{i}$ with $M_{1} \cap M_{2}=\partial M_{1}=\partial M_{2}=\partial M \times 0$. Set $D M=M_{1} \cup M_{2}$. It remains to prove that each point $(a, 0) \in M_{1} \cap M_{2}$ has a neigh- 
borhood $V$ in $D M$ such that $\left(V, V \cap M_{1}\right)$ is lipeomorphic to $\left(R^{n}, R_{+}^{n}\right)$. Choose a lipeomorphism $h: U \rightarrow R_{+}^{n}$ of an open neighborhood $U$ of $a$ in $M$. Then $V=f_{1} U \cup$ $\cup f_{2} U$ is an open neighborhood of $(a, 0)$ in $D M$. Define $p: R^{n} \rightarrow R^{n}$ by $p(x)=$ $=\left(x_{1}, \ldots, x_{n-1},-x_{n}\right)$ and $h^{*}: V \rightarrow R^{n}$ by $h^{*}\left|f_{1} U=h f_{1}^{-1}\right| f_{1} U$ and $h^{*} \mid f_{2} U=$ $=p h f_{2}^{-1} \mid f_{2} U$. Then $h^{*}$ is a homeomorphism and defines lipeomorphisms $f_{1} U \rightarrow R_{+}^{n}$ and $f_{2} U \rightarrow p R_{+}^{n}$. To prove that $h^{*}$ is a lipeomorphism it suffices to show that $f_{1} U \cup$ $\cup f_{2} U$ is a LIP proper union. Let $(b, 0) \in f_{1} U \cap f_{2} U=(U \cap \partial M) \times 0$. Choose $r>0$ with $B(b, 2 r) \subset U$. For every $x \in B(b, r) \cap$ int $M$, we have $d(x, U \cap \partial M)=d(x, \partial M)$. Using the metric $d_{1}$ of 2.39 in $M \times R^{1}$ we obtain

$$
\frac{d_{1}\left(f_{2}(x), f_{1} U \cap f_{2} U\right)}{d_{1}\left(f_{2}(x), V \backslash f_{2} U\right)}=\frac{d(x, U \cap \partial M)+d(x, \partial M)}{d(x, \partial M)}=2 .
$$

By 2.24 , the union $f_{1} U \cup f_{2} U$ is LIP proper.

\section{Embedding}

4.1. By Whitney's embedding theorem, every DIFF $n$-manifold can be DIFF embedded into $R^{2 n+1}$. Similar results hold for PL and TOP manifolds. It is therefore natural to ask whether every LIP $n$-manifold can be LIP embedded into $R^{2 n+1}$. We have not been able to solve this problem. However, we shall prove in 4.5 and 4.9 that a LIP $n$-manifold can be LIP embedded into $R^{n(n+1)}$ and that there is a locally LIP flat LIP embedding into $R^{n(n+2)}$. Before that, we prove a weaker result, which gives a new proof for the equivalence of the two definitions 3.1 and 3.3 of a LIP manifold.

4.2. Theorem. Let $M$ be a LIP $n$-manifold in the atlas sense 3.3. If $M$ has a countable basis, then $M$ is lipeomorphic to a closed subset of $R^{(n+1)^{2}}$.

Proof. For every $x \in M$, choose a chart $\left(U_{x}, h_{x}\right)$ at $x$ such that $\bar{U}_{x}$ is compact and $h_{x} U_{x}$ is open in $R_{+}^{n}$. By $[2 \mathcal{2}, 2.7]$, the cover $\left(U_{x}\right)_{x \in M}$ has a locally finite countable open refinement $\mathscr{B}$ such that $\mathscr{B}=\mathscr{B}_{0} \cup \ldots \cup \mathscr{B}_{n}$ where the members of each $\mathscr{B}_{i}$ are pairwise disjoint. Let $\mathscr{B}_{i}=\left\{U_{i 1}, U_{i 2}, \ldots\right\}$, and choose a LIP embedding $h_{i}$ of $V_{i}=\cup \mathscr{B}_{i}$ into $R_{+}^{n}$ such that for every $j, h_{i} U_{i j}$ is an open subset of $R_{+}^{n} \cap B^{n}\left(3 j e_{1}, 1\right)$. Since $M$ is normal, it has an open cover $\left\{W_{i} \mid 0 \leqq i \leqq n\right\}$ such that $\bar{W}_{i} \subset V_{i}$. From 2.5 it easily follows that there is a LIP map $\varphi_{i}: M \rightarrow I$ such that $\varphi_{i} \mid \bar{W}_{i}=1$ and spt $\varphi_{i} \subset V_{i}$. Then the product $\varphi_{i} h_{i}$, extended by zero to $M$, is LIP. Let $s=(n+1)^{2}$ and define $f: M \rightarrow R^{s}$ by $f=\left(\varphi_{0}, \varphi_{0} h_{0}, \ldots, \varphi_{n}, \varphi_{n} h_{n}\right)$. Then $f$ is LIP. We show that $f$ is the desired LIP embedding.

Since $\varphi_{i} \mid \bar{W}_{i}=1$, we have $\left|f\left(h_{i}^{-1}(x)\right)-f\left(h_{i}^{-1}(y)\right)\right| \geqq|x-y|$ for all $x, y \in h_{i} W_{i}$. Thus $f h_{i}^{-1}: h_{i} W_{i} \rightarrow f W_{i}$ is a lipeomorphism, which implies that $f$ defines a lipeomorphism of $W_{i}$ onto $f W_{i}$. Hence $f$ is a LIP immersion in the obvious sense. To 
prove that $f$ is injective, assume $f(x)=f(y)$. If $x \in W_{i}$, then $\varphi_{i}(y)=\varphi_{i}(x)=1$. Thus $y \in V_{i}$, and $h_{i}(x)=\varphi_{i}(x) h_{i}(x)=\varphi_{i}(y) h_{i}(y)=h_{i}(y)$, whence $y=x$.

We complete the proof by showing that $f$ is proper. Let $A$ be a compact set in $R^{s}$. Since $M$ is metrizable, it suffices to show that an arbitrary sequence $\left(x_{v}\right)$ in $f^{-1} A$ has a convergent subsequence. Choose a positive integer $k$ such that $A \subset B^{s}(3 k+2)$. We may assume that for some $i, x_{v} \in W_{i}$ for all $v$. Since $\varphi_{i} \mid W_{i}=1$, we have $\left|h_{i}\left(x_{v}\right)\right| \leqq\left|f\left(x_{v}\right)\right|<3 k+2$ for all $v$. Since $h_{i} U_{i j} \subset B^{n}\left(3 j e_{1}, 1\right)$, this implies $x_{v} \in U\left\{U_{i j} \mid 1 \leqq j \leqq k\right\}$ for all $v$. Since each $\bar{U}_{i j}$ is compact, $\left(x_{v}\right)$ has a convergent subsequence.

4.3. Remarks. (1) It follows from the proof of 4.2 that the map $\left(\varphi_{0} h_{0}, \ldots, \varphi_{n} h_{n}\right): M \rightarrow R^{n(n+1)}$ is a proper LIP immersion.

(2) One can show that the embedding constructed above is locally LIP flat. We omit the proof, since a better result will be given in 4.9 .

(3) Theorem 4.2 holds, with essentially the same proof, for all locally compact separable metric spaces which can be locally LIP embedded into $R^{n}$.

4.4. Lemma. Let $M$ be a LIP n-manifold (in the sense of 3.1), let $s>2 n$, let $f: M \rightarrow R^{s}$ be a LIP immersion and let $\varepsilon: M \rightarrow(0, \infty)$ be continuous. Then there is an injective LIP immersion $g: M \rightarrow R^{s}$ such that $|f(x)-g(x)|<\varepsilon(x)$ for all $x \in M$. If $f$ is injective in a neighborhood $U$ of a closed set $A$, we may choose $g|A=f| A$.

Proof. We shall give a LIP version of Milnor's proof [21, 1.29] for the corresponding DIFF result. Choose an open locally finite refinement $\left(U_{i}\right)_{i \in Z}$ of the cover $(M \backslash A, U)$ of $M$ such that $\bar{U}_{i}$ is compact and $f \mid U_{i}$ is a LIP embedding for all $i$. The indexing is chosen so that $\left\{i \mid i \leqq 0\right.$ and $\left.U_{i} \neq \emptyset\right\}=\left\{i \mid \emptyset \neq U_{i} \subset U\right\}$. Next choose an open cover $\left(V_{i}\right)_{i \in Z}$ of $M$ with $\bar{V}_{i} \subset U_{i}$ for all $i$. By 2.5 there are LIP maps $\varphi_{i}: M \rightarrow I, i>0$, such that $\varphi_{i} \mid \bar{V}_{i}=1$ and $\operatorname{spt} \varphi_{i} \subset U_{i}$.

We shall inductively construct LIP immersions $g_{j}: M \rightarrow R^{s}, j \geqq 0$, such that $g_{0}=f, g_{j}=g_{j-1}+\varphi_{j} b_{j}$, where $b_{j} \in R^{s}$ is yet to be chosen. The first requirement is that $\left|b_{j}\right|<2^{-j} \min \varepsilon \bar{U}_{j}$ (or $b_{j}=0$ if $U_{j}=\emptyset$ ). Then $\left|g_{j}(x)-g_{j-1}(x)\right|<2^{-j} \varepsilon(x)$ for all $x \in M$. Choose an open cover $\left\{B_{1}, \ldots, B_{r}\right\}$ of $\bar{U}_{j}$ and a positive number $l$ such that $\left|g_{j-1}(x)-g_{j-1}(y)\right| \geqq l d(x, y)$ for all $x, y$ in $B_{k}, 1 \leqq k \leqq r$. The second requirement is that $\left|b_{j}\right|<l / 2 \operatorname{lip}\left(\varphi_{j} \mid \bar{U}_{j}\right)$. Then $\left|g_{j}(x)-g_{j}(y)\right| \geqq\left|g_{j-1}(x)-g_{j-1}(y)\right|-$ $-\left|\varphi_{j}(x)-\varphi_{j}(y)\right|\left|b_{j}\right| \geqq 2^{-1} l d(x, y)$ for all $x, y$ in $U_{j} \cap B_{k}, 1 \leqq k \leqq r$. Since $g_{j}(x)=$ $=g_{j-1}(x)$ outside spt $\varphi_{j}$, it follows that $g_{j}$ is a LIP immersion. Finally, let $N$ be the open set in $M \times M$ consisting of pairs $(x, y)$ such that $\varphi_{j}(x) \neq \varphi_{j}(y)$. Define a LIP map $\psi: N \rightarrow R^{s}$ by $\psi(x, y)=\left(g_{j-1}(y)-g_{j-1}(x)\right) /\left(\varphi_{j}(x)-\varphi_{j}(y)\right)$. Since $M \times M$ is a LIP $2 n$-manifold and $s>2 n$, the set $\psi N$ is of Hausdorff $s$-measure zero (see 6.2). Hence we may require $b_{j} \notin \psi N$. Then $g_{j}(x)=g_{j}(y)$ if and only if $\varphi_{j}(x)=\varphi_{j}(y)$ and $g_{j-1}(x)=g_{j-1}(y)$.

Define $g: M \rightarrow R^{s}$ by $g(x)=\lim _{j \rightarrow \infty} g_{j}(x)$. For each $x_{0} \in M$, there is a neighborhood $V$ of $x_{0}$ and an integer $j$ such that $g(x)=g_{j}(x)$ for $x \in V$. Hence $g$ is a 
LIP immersion. Furthermore, $|f(x)-g(x)|<\varepsilon(x)$ for all $x \in M$, and $g|A=f| A$. It remains to prove that $g$ is injective. Suppose that $g(x)=g(y)$ with $x \neq y$. Then $\varphi_{j}(x)=\varphi_{j}(y)$ and $g_{j-1}(x)=g_{j-1}(y)$ for all $j>0$. For $j=1$ this yields $f(x)=f(y)$. Hence $x$ and $y$ cannot be in the same set $U_{i}$. If $x \in V_{i}$ for some $i>0$, then $\varphi_{i}(y)=$ $=\varphi_{i}(x)=1$, and thus $y \in U_{i}$. Hence $x$ and $y$ are in $U$. This is impossible, since $f \mid U$ is injective.

4.5. Theorem. Let $M$ be a LIP n-manifold. Then there is a closed LIP embedding $f: M \rightarrow R^{n(n+1)}$.

Proof. For $n=1$ the theorem follows from 3.11. Assume $n \geqq 2$. By 4.3 (1), there is a proper LIP immersion $F: M \rightarrow R^{n(n+1)}$. Since $n(n+1)>2 n$, it follows from 4.4 that there is an injective LIP immersion $f: M \rightarrow R^{n(n+1)}$ such that $|f(x)-F(x)|<1$ for all $x \in M$. Then $f$ is a proper map and hence a closed LIP embedding.

4.6. Remark. Lemma 4.4 and Theorem 4.5 can be generalized for locally compact separable metric spaces which can be locally LIP embedded into $R^{n}, n \geqq 2$, see Remark 4.3 (3).

4.7. Theorem. Let $D$ be a LIP $k$-ball in $R^{n}$. Then there is a strong lipeomorphism $\varphi$ of $R^{n+k}=R^{n} \times R^{k}$ onto itself such that $\varphi D=I^{k}$.

Proof. This is the LIP version of a theorem of Klee [26, Theorem 2.5.1, p. 74]. Using 5.6 instead of Tietze's theorem, all maps occurring in the proof can be made Lipschitz.

4.8. Corollary. If $M$ is a LIP $k$-submanifold of $R^{n}, M$ is locally LIP flat in $R^{n+k}=R^{n} \times R^{k}$.

4.9. Theorem. If $M$ is a LIP n-manifold, there is a closed locally LIP flat LIP embedding $f: M \rightarrow R^{n(n+2)}$.

Proof. This follows from 4.5 and 4.8 .

4.10. Remark. Theorem 4.9 is one dimension better than the result announced in 4.3 (2).

4.11. Projections. Suppose that $f: M \rightarrow R^{s}$ is a LIP embedding of a LIP $n$-manifold $M, s>2 n+1$. One might think that the dimension of the target space could be lowered by choosing a suitable projection of $R^{s}$ onto an $(s-1)$-dimensional linear subspace. In the DIFF category this is possible, see [7, Théorème 5, p. 12]. Set $N=f M$, and let $\sigma(N)=\{(x-y) /|x-y| \mid x, y \in N, x \neq y\} \subset S^{s-1}$ be the set of directions of all secants of $N$. It is easy to see that the Hausdorff $(s-1)$-measure of $\sigma(M)$ is zero. Hence there is $a \in S^{s-1} \backslash \sigma(N)$. Let $p$ be a projection of $R^{s}$ in the direction $a$ onto an $(s-1)$-dimensional subspace $V$. Then $p \mid N$ is injective and Lipschitz. Continuing similarly we obtain a LIP injection of $N$ into $R^{2 n+1}$. How- 
ever, $p \mid N$ need not be a LIP embedding. In fact, we shall next construct a strongly LIP flat arc $A \subset R^{s}$ such that $\overline{\sigma(U)}=S^{s-1}$ for every neighborhood $U$ of a point in $A$. It is clear that no projection of $R^{s}$ onto a proper subspace can define a LIP immersion of $A$.

For $s=1$ take $A=I^{1}$. For $s=2$ see 3.10 (4). Let $s \geqq 3$, and let $(r, \varphi, z)$ be the cylindrical coordinates in $R^{s}$. Thus $x_{1}=r \cos \varphi, x_{2}=r \sin \varphi$, and $\left(x_{3}, \ldots, x_{s}\right)=$ $=\left(z_{1}, \ldots, z_{s-2}\right)$. Define $g: R^{s} \rightarrow R^{s}$ by $g(r, \varphi, z)=(r, \varphi+\log |x|, z)$. It is easy to verify that $g$ is an $L$-lipeomorphism for some $L$. For example, computing the derivative $g^{\prime}(x)$ shows that it is bounded for $x \neq 0$. Moreover, $g^{-1}(r, \varphi, z)=(r, \varphi-\log |x|, z)$. Choose a countable dense set $\left\{a_{j} \mid j \in \mathbf{N}\right\}$ in $\left\{x \in S^{s-1} \mid x_{1}=0\right\}$. For each $j \in \mathbf{N}$ choose a rotation $h_{j}$ of $R^{s}$ such that $h_{j}\left(e_{1}\right)=e_{1}, h_{j}\left(e_{2}\right)=a_{j}$. Set $f_{j}=h_{j} g h_{j}^{-1}$ and observe that $f_{j} \mid S^{s-1}\left(e^{2 \pi k}\right)=\mathrm{id}$ for all integers $k$. Hence we can define a homeomorphism $f: R^{s} \rightarrow R^{s}$ by $f(x)=f_{j}(x)$ for $e^{-2 j \pi} \leqq|x| \leqq e^{-2(j-1) \pi}, j \in \mathbf{N}$, and $f(x)=x$ for $x=0$ and $|x| \geqq 1$. By $2.35, f \mid R^{s} \backslash 0$ is an L-lipeomorphism. Hence $f$ is an L-lipeomorphism. Let $J$ be the segment $\left\{t e_{2} \mid-1 \leqq t \leqq 1\right\}$. Then $A=f J$ is a strongly LIP flat (in the obvious sense) arc in $R^{s}$ consisting of two "twisted" logarithmic spirals and the origin. Clearly $\operatorname{cl} \sigma\left(A \cap B^{s}(\varepsilon)\right)=S^{s-1}$ for all $\varepsilon>0$.

4.12. Non-manifolds. Every finite-dimensional separable metric space can be topologically embedded into a euclidean space, see [14, Theorem V3, p. 60]. We shall next show that the corresponding result is not true for LIP embeddings by constructing a countable metric space which cannot be LIP embedded into any euclidean space.

Let $X$ be the set consisting of all positive integers and the point $\infty$. Let $a(1)>$ $>a(2)>\ldots$ be a sequence such that $a(i) \rightarrow 0$ and the series $\sum_{i} a(i)^{n}$ diverges for every $n \in \mathbf{N}$. For example, we may choose $a(i)=1 / \log (i+1)$. Define a metric $d$ in $X$ by $d(i, j)=a(\min (i, j))$ if $i \neq j$ and by $d(i, i)=0$. Then $X$ has its usual topology and is compact. Suppose that $f: X \rightarrow R^{n}$ is a LIP embedding. Then there is $q>0$ such that $|f(i)-f(j)| \geqq q d(i, j)$ for all $i, j$ in $X$. Since $d(i, j) \geqq a(i)$ whenever $i, j \in X \backslash \infty$ and $i \neq j$, it follows that the balls $B_{i}=B^{n}(f(i), q a(i) / 2)$ are disjoint for $i \in X \backslash \infty$. Since the series $\sum_{i} a(i)^{n}$ diverges, the set $E=\cup\left\{B_{i} \mid i \in X \backslash \infty\right\}$ has an infinite measure. On the other hand, since $f X$ is compact, $E$ is bounded, and we obtain a contradiction.

Observe that we did not make use of the fact that $f$ is LIP. Hence there is no homeomorphism $f: X \rightarrow Y \subset R^{n}$ such that $f^{-1}$ is LIP.

\section{Extension and approximation}

5.1. The main results of this section deal with maps of a metric space $X$ into a LIP manifold $M$. In 5.12 we show that if $A$ is closed in $X$, every LIP map $f: A \rightarrow M$ has a LIP extension to a neighborhood of $A$ in $X$. This result will be used in 5.18 to prove that every map $f: X \rightarrow M$ can be approximated by a LIP map. 
5.2. Definition. A LIP partition of unity subordinated to an open cover $\left(U_{j}\right)_{j \in J}$ of a metric space $X$ is family $\left(\varphi_{j}\right)_{j \in J}$ of LIP maps $\varphi_{j}: X \rightarrow I$ such that the supports $\operatorname{spt} \varphi_{j}=\mathrm{cl} \varphi_{j}^{-1}(0,1]$ form a locally finite family, spt $\varphi_{j} \subset U_{j}$ for all $j \in J$, and $\sum_{j \in J} \varphi_{j}(x)=1$ for all $x \in X$.

5.3. Theorem. Let $\left(U_{j}\right)_{j \in J}$ be an open cover of a metric space $X$. Then there is a LIP partition of unity subordinated to this cover.

Proof. We may assume that $U_{j} \neq X$ for all $j$. Choose a locally finite open refinement $\left(V_{j}\right)_{j \in J}$ of $\left(U_{j}\right)_{j \in J}$ such that $\bar{V}_{j} \subset U_{j}$ [8, p. 162]. Set $\psi_{j}(x)=d\left(x, \mathbf{C} V_{j}\right)$ and $\varphi_{j}=\psi_{j} / \sum_{j \in J} \psi_{j}$. Then spt $\varphi_{j} \subset \bar{V}_{j}$, and the theorem follows.

5.4. Theorem. Let $X$ be a metric space and let $f_{0}, f_{1}$ be real-valued functions on $X$ such that $f_{0}$ is upper semicontinuous, $f_{1}$ is lower semicontinuous, and $f_{0}(x)<f_{1}(x)$ for all $x \in X$. Then there is a LIP map $g: X \rightarrow R^{1}$ such that $f_{0}(x)<g(x)<f_{1}(x)$ for all $x \in X$.

Proof. The proof given in $[8,4.3, \mathrm{p}$. 171] for the paracompact case yields a LIP map $g$ if a LIP partition of unity is used.

5.5. A metric space $X$ is an absolute LIP extensor (ALE) if for every closed set $B$ in every metric space $Y$, every LIP map $f: B \rightarrow X$ has a LIP extension to $Y$. If every such $f$ has a LIP extension to a neighborhood of $B, X$ is an absolute LIP neighborhood extensor (ALNE). Our next goal is to show that every LIP manifold is an ALNE. The proof is based on LIP versions of Tietze's theorem, due to McShane, and Hanner's theorem, which states that being an ALNE is a local property.

5.6. Lemma. Let $A$ be a subset of a metric space $X$ and let $f: A \rightarrow R^{n}$ be Lipschitz. Then $f$ has a Lipschitz extension $g: X \rightarrow R^{n}$ with $\operatorname{lip} g \leqq n^{1 / 2} \operatorname{lip} f$.

Proof. Apply McShane [19, Theorem 1] to each coordinate map of $f$.

5.7. Theorem. $R^{n}$ and $R_{+}^{n}$ are ALE's.

Proof. Since $R_{+}^{n}$ is a LIP retract of $R^{n}$, it suffices to show that $R^{n}$ is an ALE. Let $B$ be closed in $Y$, and let $f: B \rightarrow R^{n}$ be LIP. For each $b \in B$ choose an open neighborhood $U_{b}$ in $Y$ such that $f \mid U_{b} \cap B$ is Lipschitz. By 5.6, $f \mid U_{b} \cap B$ has a LIP extension $f_{b}: Y \rightarrow R^{n}$. Let $\left(\varphi_{i}\right)_{i \in I}$ be a LIP partition of unity subordinated to the cover $\{Y \backslash B\} \cup\left\{U_{b} \mid b \in B\right\}$ of $Y$. Set $J=\left\{j \in I \mid B \cap \operatorname{spt} \varphi_{j} \neq \emptyset\right\}$. For each $j \in J$ choose $b \in B$ with spt $\varphi_{j} \subset U_{b}$ and set $g_{j}=f_{b}$. Then $g(x)=\sum_{j \in J} \varphi_{j}(x) g_{j}(x)$ defines a LIP map $g: X \rightarrow R^{n}$. If $x \in B$ and $\varphi_{i}(x)>0$, then $i \in J$ and $g_{i}(x)=f(x)$, whence $g(x)=f(x)$.

5.8. Lemma. Let $\mathscr{U}$ be an open cover of a metric space $X$ satisfying the following conditions:

(1) If $U \in \mathscr{U}$ and $V$ is open in $U$, then $V \in \mathscr{U}$.

(2) If $U$ and $V$ are in $\mathscr{U}$, then $U \cup V \in \mathscr{U}$. 
(3) If $\left\{U_{i} \mid i \in I\right\}$ is a pairwise disjoint subfamily of $\mathscr{U}$, then $U\left\{U_{i} \mid i \in I\right\} \in \mathscr{U}$. Then $\mathscr{U}$ contains all open sets of $X$.

Proof. This follows directly from Michael [20, 2.1 (e) and 3.3].

5.9. We shall give a general version of Hanner's theorem and obtain the LIP result as a special case. Let $F$ be a class of maps between metric spaces. We say that a metric space $X$ is an $\mathrm{ANE}_{F}$ if for every closed set $B$ in every metric space $Y$, every $F$-map $f: B \rightarrow X$ has an $F$-extension $g: U \rightarrow X$ to an open neighborhood $U$ of $B$. Thus ALNE means $\mathrm{ANE}_{F}$ for $F=\mathrm{LIP}$.

5.10. Theorem. Let $F$ be a class of maps between metric spaces such that the following conditions are satisfied:

(1) If $f: Y \rightarrow X$ is in $F$ and if $B$ is open or closed in $Y$, then $f \mid B: B \rightarrow X$ is in $F$.

(2) Let $A$ be open in $X$, let $j: A \rightarrow X$ be the inclusion, and let $f: Y \rightarrow A$. Then $f \in F$ if and only if $j f \in F$.

(3) If $f: Y \rightarrow X$ is a map such that every point in $Y$ has a neighborhood $U$ such that $f \mid U \in F$, then $f \in F$.

Suppose that $X$ is a metric space such that every point in $X$ has a neighborhood which is an $\mathrm{ANE}_{F}$. Then $X$ is an $\mathrm{ANE}_{F}$.

Proof. The proof of Hanner [13, 3.1] shows that every open subset of an $\mathrm{ANE}_{F}$ is an $\mathrm{ANE}_{F}$. Hence every point of $X$ has an open neighborhood which is an $\mathrm{ANE}_{F}$. Let $\mathscr{U}$ be the family of all open subsets of $X$ which are $\mathrm{ANE}_{F}$ 's. Then $\mathscr{U}$ is an open cover of $X$. It suffices to show that $\mathscr{U}$ satisfies the conditions of 5.8. The condition (1) follows from the first remark of the present proof. The condition (3) is proved exactly as in Michael [20, 4.1 (c)]. To prove (2), we can follow Hanner's proof $[13,3.3$, a)] with a slight modification. Indeed, the maps $g$ and $F$ constructed in the proof need not be in $F$. To arrange this, we choose the sets $Y_{1}, Y_{2}$ so that $\bar{Y}_{1} \cap \bar{Y}_{2}=\emptyset$. Then choose open neighborhoods $V_{i}$ of $\bar{Y}_{i}$ such that $\bar{V}_{1} \cap \bar{V}_{2}=\emptyset$. Let $V_{0}=Y \backslash\left(V_{1} \cup V_{2}\right)$. Then $g \mid\left(U_{0} \cap V_{0}\right) \cup B$ is in $F$, since it is locally in $F$. Replacing $Y_{i}$ by $V_{i}$ we may thus assume that $g$ is in $F$. To show that the map $F$ is in the family $F$, we observe that the sets $W_{1}=U_{1} \backslash \bar{Y}_{2}$ and $W_{2}=U_{2} \backslash \bar{Y}_{1}$ form an open cover of $U$ and $F\left|W_{i}=g_{i}\right| W_{i}, i=1,2$.

5.11. Corollary. Let $X$ be a metric space such that every point has a neighborhood which is an ALNE. Then $X$ is an ALNE.

5.12. Theorem. Every LIP manifold is an ALNE.

Proof. Let $M$ be a LIP manifold. Every point in $M$ has a neighborhood $U$ lipeomorphic to $R^{n}$ or $R_{+}^{n}$. Since the property ALE is obviously a LIP invariant, it follows from 5.7 that $U$ is an ALE and hence an ALNE. By 5.11, $M$ is an ALNE.

5.13. Theorem. Let $M$ be a LIP submanifold of $R^{n}$. Then $M$ is a LIP neighborhood retract of $R^{n}$. 
Proof. Since $M$ is locally compact, it has an open neighborhood $U$ such that $M$ is closed in $U$. By 5.12 , the identity map $M \rightarrow M$ has a LIP extension to an open neighborhood $V$ of $M$ in $U$.

5.14. Approximation. Suppose that $f: X \rightarrow Y$ is a map between metric spaces $X, Y$. It is natural to ask whether $f$ can be approximated in some sense by LIP maps. If $Y$ is too general, the answer is negative, as is seen from the following counterexample: Let $X=[-1,1]$, let $f(x)=(x, x \sin (1 / x)), f(0)=(0,0)$, and let $Y=\operatorname{im} f \subset$ $\subset R^{2}$. Then $Y$ is an arc which is not locally rect fiable at 0 . If $g: X \rightarrow Y$ is LIP, then im $g$ lies entirely either in the left half plane or in the right half plane. Thus $f$ cannot be approximated by LIP maps.

We shall show that the approximation is always possible if $Y$ is a LIP manifold. Moreover, the approximating map can be obtained from $f$ by a small homotopy. A relative version will also be given.

5.15. Function spaces. Given two metric spaces $X, Y$ we let $T(X, Y)$ denote the set of all maps $f: X \rightarrow Y$. We shall use the majorant topology in $T(X, Y)$. A basis for this topology consists of sets $U(f, \varepsilon)=\left\{g \mid d^{\prime}(f(x), g(x))<\varepsilon(x)\right.$ for all $\left.x \in X\right\}$. Here $d^{\prime}$ is the metric of $Y, f \in T(X, Y)$, and $\varepsilon: X \rightarrow(0, \infty)$ is an arbitrary map. This topology is equal to the graph topology (Whitehead [31, (5.2), p. 172]), whose basis consists of sets $W_{U}=\{f \mid \Gamma(f) \subset U\}$. Here $\Gamma(f)$ is the graph of $f: X \rightarrow Y$ and $U$ is a arbitrary open set in $X \times Y$. Hence the topology of $T(X, Y)$ is independent of $d^{\prime}$.

The elements of $U(f, \varepsilon)$ are called e-approximations of $f$. A homotopy $h: X \times I \rightarrow Y$ is called an $\varepsilon$-homotopy if $h_{t}$ is an $\varepsilon$-approximation of $h_{0}$ for every $t \in I$.

Let $X, Y, Z$ be metric spaces, and let $\varphi: Y \rightarrow Z$ be a map. Then $\varphi$ induces a function $\varphi_{*}: T(X, Y) \rightarrow T(X, Z)$ by $\varphi_{*}(f)=\varphi f$. Using the graph topology, it is easy to give a straightforward proof for the following result:

5.16. Lemma. $\varphi_{*}: T(X, Y) \rightarrow T(X, Z)$ is continuous.

5.17. Theorem. Let $M$ be a LIP manifold, $X$ a metric space, $f: X \rightarrow M$ continuous, $\varepsilon: X \rightarrow(0, \infty)$ continuous, and $A \subset X$ closed. Then there is a continuous $\delta: A \rightarrow(0, \infty)$ such that if $g: A \rightarrow M$ is LIP and a $\delta$-approximation to $f \mid A$, then $g$ has a LIP extension $u: X \rightarrow M$ which is $\varepsilon$-homotopic to $f$. Moreover, if $f \mid A$ is LIP and $g=f \mid A$, the homotopy can be chosen to be fixed on $A$.

Proof. Special case: $M$ is an open subset $W$ of $R^{n}$. We may assume that $\varepsilon(x)<$ $<d\left(f(x), R^{n} \backslash W\right)$ for all $x \in X$. We show that $\delta=\varepsilon \mid A$ satisfies the conditions of the theorem.

So let $g: A \rightarrow W$ be a LIP map, which is an $\varepsilon \mid A$-approximation to $f \mid A$. For every $x \in X$ choose an open neighborhood $U_{x}$ as follows: If $x \in X \backslash A$, then $U_{x} \subset$ $\subset X \backslash A$ and $d\left(f U_{x}\right)<\inf \varepsilon U_{x}$. If $x \in A$, then $g \mid U_{x} \cap A$ is Lipschitz. By 5.6, we 
can choose a LIP extension $g_{x}: X \rightarrow R^{n}$ of $g \mid U_{x} \cap A$. We may assume, replacing $U_{x}$ by a smaller neighborhood, that $\left|g_{x}(y)-f(y)\right|<\varepsilon(y)$ for all $y \in U_{x}$ and for all $x \in A$. Let $\left(\varphi_{x}\right)_{x \in X}$ be a LIP partition of unity subordinated to the cover $\left(U_{x}\right)_{x \in X}$. For each $x \in X$ define $u_{x}: X \rightarrow R^{n}$ by $u_{x}=g_{x}$ if $x \in A$ and by $u_{x}(y)=f(x)$ if $x \in X \backslash A$ and $y \in X$. Set $u=\sum_{x \in X} \varphi_{x} u_{x}$. Then $u$ is clearly LIP. If $a \in A$ and $\varphi_{x}(a)>0$, then $u_{x}(a)=g_{x}(a)=g(a)$, whence $u(a)=g(a)$. If $y \in X$ and $\varphi_{x}(y)>0$, then $\left|u_{x}(y)-f(y)\right|<\varepsilon(y)$,w hence $|u(y)-f(y)|<\varepsilon(y)$. Setting $h(x, t)=(1-t) f(x)+$ $+t u(x)$ we obtain an $\varepsilon$-homotopy from $f$ to $u$. Since $\varepsilon(x)<d\left(f(x), R^{n} \backslash W\right)$, im $h \subset W$. If $g=f \mid A, h$ is fixed on $A$. The special case is proved.

General case. By 4.2, there is a LIP embedding of $M$ into a euclidean space $R^{n}$ for some $n$. From 5.16 it follows that we may assume $M \subset R^{n}$. By 5.13, there is a LIP retraction $r: W \rightarrow M$ of an open neighborhood $W$ of $M$. By 5.16, there is a continuous $\delta: X \rightarrow(0, \infty)$ such that if $v: X \rightarrow W$ is a $\delta$-approximation to $f$, then $r v: X \rightarrow M$ is an $\varepsilon$-approximation to $r f=f$. We may assume $\delta(x)<d\left(f(x), R^{n} \backslash W\right)$ for all $x \in X$. Let $g: A \rightarrow M$ be LIP and a $\delta \mid A$-approximation to $f \mid A$. By the special case, $g$ has a LIP extension $v: X \rightarrow W$ which is $\delta$-homotopic to $f$ in $W$. Then $u=r v: X \rightarrow M$ is a LIP extension of $g$. If $h: X \times I \rightarrow W$ is a $\delta$-homotopy from $f$ to $v$, then $r h$ is an $\varepsilon$-homotopy from $f$ to $u$ in $M$. If $g=f \mid A$ and if $h$ is fixed on $A$, then also, $r h$ is fixed on $A$.

5.18. Corollary. Let $M$ be a LIP manifold, $X$ a metric space, $f: X \rightarrow M$ continuous, and $\varepsilon: X \rightarrow(0, \infty)$ continuous. Then there is a LIP map $g: X \rightarrow M$ which is $\varepsilon$-homotopic to $f$. Moreover, if $f \mid A$ is LIP for a closed set $A \subset X$, the homotopy can be chosen to be fixed on $A$.

\section{General position}

6.1. General position is an important tool in PL topology. A typical example is the following result: Let $P$ and $Q$ be compact polyhedra of dimensions $p$ and $q$ in the interior of a PL $n$-manifold $M$. If $p+q \leqq n-1$, there is for every $\varepsilon>0$ a PL homeomorphism $h: M \rightarrow M$ such that $d(h$, id $)<\varepsilon$ and $P \cap h Q=\emptyset$. In this section we try to find LIP analogues of results like this. It turns out that on a LIP manifold, polyhedral conditions can often be replaced by assumptions concerning rectifiability and Hausdorff measure. For example, we shall prove a LIP version of the above result, assuming that $P$ is $p$-rectifiable and $Q$ is of Hausdorff $(q+1)$ measure zero.

6.2. Hausdorff measure and rectifiability. An excellent reference on these topics is Federer [10], and we shall use his terminology and notation. Thus we let $\mathscr{H}^{q}(X)$, $q \geqq 0$, denote the $q$-dimensional Hausdorff measure [10, p. 171] of a separable metric space $X$. Recall that $\mathscr{H}^{0}$ is the counting measure. If $f$ is an $L$-Lipschitz map of $X$, then $\mathscr{H}^{q}(f X) \leqq L^{q} \mathscr{H}^{q}(X)$. Hence the following properties are LIP invariants of 
a separable metric space $X:$ (1) $\mathscr{H}^{q}(X)=0,(2) \mathscr{H}^{q}(X)$ is $\sigma$-finite, (3) the Hausdorff dimension of $X$ is $q$. For a compact space $X$, the property $\mathscr{H}^{q}(X)<\infty$ is also a LIP invariant.

A metric space $X$ is p-rectifiable if there is a bounded set $F \subset R^{p}$ and a Lipschitz map of $F$ onto $X$. If $X$ is a countable union of $p$-rectifiable sets, it is countably p-rectifiable. Equivalently, $X$ is countably $p$-rectifiable if there is a set $F \subset R^{p}$ and a LIP map of $F$ onto $X$. Thus countable $p$-rectifiability is a LIP invariant property.

We shall use the phrase " $\mu$ almost all" in its usual sense, meaning all except for a set of $\mu$ measure zero, and we may omit $\mu$ if it is the ordinary Lebesgue measure $\left(\mathscr{H}^{n}\right.$ in $\left.R^{n}\right)$. Following Federer [10], we do not make a distinction between measure and outer measure.

6.3. Lemma. Suppose that $A \subset R^{p}$ with $\mathscr{H}^{p}(A)<\infty, \alpha: A \rightarrow R^{n}$ is Lipschitz, $E \subset R^{n}$, and $g: E \times A \rightarrow R^{n}$ is a map such that $\varphi(x, y)=(g(x, y), y)$ defines a Lipschitz embedding $\varphi: E \times A \rightarrow R^{n} \times A$. Suppose also that $Q \subset R^{n}$ with $\mathscr{H}^{q}(Q)<\infty$ and that $0 \leqq k \leqq p+q$. Then $\mathscr{H}^{k}\left(Q \cap\left(\alpha+g_{x}\right) A\right)<\infty$ for $\mathscr{H}^{p+q-k}$ almost all $x \in E$, where $g_{x}(y)=g(x, y)$. In particular, if $p+q \leqq n$, then $Q \cap\left(\alpha+g_{x}\right) A$ is finite for almost all $x \in E$.

Proof. Setting $\psi(x, y)=\varphi(x, y)+(\alpha(y), 0)$ we obtain another Lipschitz embedding $\psi: E \times A \rightarrow R^{n} \times A$. Indeed, if $\varphi_{1}: \operatorname{im} \varphi \rightarrow E \times A$ is the inverse of $\varphi$, then $\psi_{1}(x, y)=\varphi_{1}(x-\alpha(y), y)$ defines a Lipschitz inverse $\psi_{1}$ : $\operatorname{im} \psi \rightarrow E \times A$ of $\psi$. By $[10,2.10 .45], \mathscr{H}^{p+q}(Q \times A)<\infty$. Hence the set $Q_{1}=\psi^{-1}[Q \times A]$ is of finite $\mathscr{H}^{p+q}$ measure. For $x \in R^{n}$ set $D(x)=\left\{y \in R^{p} \mid(x, y) \in Q_{1}\right\}$. Applying [10, 2.10.27] with the substitution $Y \mapsto R^{n}, Z \mapsto A, A \mapsto Q_{1}, m \mapsto p+q-k$ yields $\mathscr{H}^{k}(D(x))<\infty$ for $\mathscr{H}^{p+q-k}$ almost all $x \in R^{n}$. If $\alpha(y)+g(x, y)=z \in Q$, then $(z, y)=\psi(x, y)$ and $y \in D(x)$. Hence $Q \cap\left(\alpha+g_{x}\right) A \subset p_{1} \psi[x \times D(x)]$ where $p_{1}: R^{n} \times A \rightarrow R^{n}$ is the projection. The lemma follows.

6.4. Lemma. Suppose that $A \subset R^{p}, \alpha: A \rightarrow R^{n}$ is LIP, $E \subset R^{n}$, and $g: E \times A \rightarrow R^{n}$ is a map such that $\varphi(x, y)=(g(x, y), y)$ defines a LIP embedding $\varphi: E \times A \rightarrow R^{n} \times A$. Suppose also that $Q \subset R^{n}$ with $\mathscr{H}^{q}(Q)=0$ and that $0 \leqq k \leqq p+q$. Then $\mathscr{H}^{k}\left(Q \cap\left(\alpha+g_{x}\right) A\right)=0$ for $\mathscr{H}^{p+q-k}$ almost all $x \in E$. In particular, if $p+q \leqq n$, then $Q \cap\left(\alpha+g_{x}\right) A=\emptyset$ for almost all $x \in E$.

Proof. A slight modification of the proof of 6.3 .

6.5. Theorem. Suppose that $P \subset R^{n}$ is countably p-rectifiable and $Q \subset R^{n}$ with $\mathscr{H}^{q}(Q)=0$. If $p+q \leqq n$, then $Q \cap(P+x)=\emptyset$ for almost all $x \in R^{n}$.

Proof. Apply 6.4 with $E=R^{n}$ and $g(x, y)=x$.

6.6. Notation. We let $\|x\|=\max \left(\left|x_{1}\right|, \ldots,\left|x_{n}\right|\right)$ denote the Banach norm of a vector $x \in R^{n}$. For $z \in \operatorname{int} I^{n}$, let $w_{z}: I^{n} \rightarrow I^{n}$ be the PL homeomorphism defined by $w_{z}(x)=x+(1-\|x\|) z$. Thus $w_{z}$ is the $z$-cone extension of $\mathrm{id} \mid \partial I^{n}$ to $I^{n}=0\left(\partial I^{n}\right)$. 
6.7. Lemma. Let $P \subset \operatorname{int} I^{n}$ be countably p-rectifiable and let $Q \subset \operatorname{int} I^{n}$ with $\mathscr{H}^{q}(Q)=0$. If $p+q \geqq n$, then $\mathscr{H}^{p+q-n}\left(Q \cap w_{z} P\right)=0$ for almost all $z \in \operatorname{int} I^{n}$. If $p+q \leqq n$, then $Q \cap w_{z} P=\emptyset$ for almost all $z \in \operatorname{int} I^{n}$.

Proof. Choose a LIP map $\alpha$ of a set $A \subset R^{p}$ onto $P$. Set $E=\operatorname{int} I^{n}$ and define $g: E \times A \rightarrow E$ by $g(z, y)=(1-\|\alpha(y)\|) z$. Then $\varphi(z, y)=(g(z, y), y)$ defines a LIP embedding $\varphi: E \times A \rightarrow R^{n} \times A$, since it has a LIP inverse $\varphi_{1}: \operatorname{im} \varphi \rightarrow E \times A$, defined by $\varphi_{1}(z, y)=(z /(1-\|\alpha(y)\|), y)$. Since $\alpha+g_{z}=w_{z} \alpha$, the lemma follows from 6.4 .

6.8. Definition. A LIP isotopy of a metric space $X$ is a level preserving lipeomorphism $F: X \times I \rightarrow X \times I$ such that $F_{0}=$ id. Here we use the customary notation $F(x, t)=\left(F_{t}(x), t\right)$. If $d\left(F_{t}(x), x\right)<\varepsilon$ for all $x \in X$ and $t \in I, F$ is said to be an $\varepsilon$-isotopy. If $F \mid(X \backslash U) \times I=\mathrm{id}, F$ is supported by $U$.

6.9. Theorem. Let $M$ be a LIP n-manifold, let $P \subset$ int $M$ be compact and countably p-rectifiable, let $Q \subset M$ with $\mathscr{H}^{q}(Q)=0$, let $U$ be a neighborhood of $P \cap \bar{Q}$, and let $\varepsilon>0$. Then there is a LIP $\varepsilon$-isotopy $F$ of $M$ supported by $U$ such that $\mathscr{H}^{p+q-n}\left(Q \cap F_{1} P\right)=0$. For $p+q \leqq n$ this means $Q \cap F_{1} P=\emptyset$. If $M$ is a PL manifold, $F$ can be chosen to be a PL isotopy.

Proof. Choose LIP $n$-balls $B_{1}, \ldots, B_{k}$ in $U$ so that $P \cap \bar{Q} \subset \cup$ int $\left.B_{i} \mid 1 \leqq i \leqq k\right\}$. Choose lipeomorphisms $\varphi_{i}: B_{i} \rightarrow I^{n}$ and set $P_{1}=\varphi_{1}\left[P \cap \operatorname{int} B_{1}\right], Q_{1}=\varphi_{1}\left[Q \cap \operatorname{int} B_{1}\right]$. By 6.7, there is $z_{1} \in$ int $I^{n}$ such that $\mathscr{H}^{p+q-n}\left(Q_{1} \cap w_{z_{1}} P_{1}\right)=0$. The Alexander trick $\left[25\right.$, p. 37] gives a PL isotopy of $I^{n}$ fixed on $\partial I^{n}$ and finishing with $w_{z_{1}}$. With the aid of $\varphi_{1}$, we can transfer this isotopy to a LIP isotopy of $B_{1}$. Extending this isotopy by the identity, we obtain a LIP isotopy $F^{1}$ of $M$ supported by $B_{1}$ such that $\mathscr{H}^{p+q-n}\left(Q \cap F_{1}^{1} P \cap\right.$ int $\left.B_{1}\right)=0$. Setting $P_{2}=\varphi_{2}\left[F_{1}^{1} P \cap \operatorname{int} B_{2}\right], Q_{2}=\varphi_{2}\left[Q \cap\right.$ int $\left.B_{2}\right]$ we similarly choose $z_{2}$ with $\mathscr{H}^{p+q-n}\left(Q_{2} \cap w_{z_{2}} P_{2}\right)=0$ and obtain a LIP isotopy $F^{2}$ of $M$ supported by $B_{2}$ with $\mathscr{H}^{p+q-n}\left(Q \cap F_{1}^{2} F_{1}^{1} P \cap\left(\right.\right.$ int $B_{1}$ vint $\left.\left.B_{2}\right)\right)=0$. After $k$ steps we have a LIP isotopy $F=F^{k} \ldots F^{1}$ of $M$ supported by $U$. such that $\mathscr{H}^{p+q-n}\left(Q \cap F_{1} P\right)=0$. Since the points $z_{i}$ can be chosen to be arbitrarily close to the origin, $F$ can be chosen to be an $\varepsilon$-isotopy. If $M$ is PL, all maps can be chosen to be PL.

6.10. Theorem. The condition $\mathscr{H}^{p+q-n}\left(Q \cap F_{1} P\right)=0$ of 6.9 can be replaced by $\mathscr{H}^{p+q-n}\left(P \cap F_{1} Q\right)=0$.

Proof. Replace the isotopy $F$ by its inverse $F^{-1}: M \times I \rightarrow M \times I$.

6.11. Remark. There are several obvious modifications of 6.9. We may use 6.3 instead of 6.4 and obtain a finiteness condition on $\mathscr{H}^{p+q-n}\left(Q \cap F_{1} P\right)$ instead of the zero condition. For example, if $P^{p}$ and $Q^{q}$ are compact LIP submanifolds of $M^{n}$ with $p+q \leqq n$, then there is a small LIP isotopy of $M$ which carries $P$ onto $P^{r}$ with $Q \cap P^{\prime}$ finite. 
6.12. Applications. We shall give three applications of LIP general position. First, we show that removing a set of sufficiently small Hausdorff dimension does not change the lowdimensional homotopy groups of a LIP manifold. Next, we show that for a compact set $X \subset R^{n}, \mathscr{H}^{q+1}(X)=0$ implies dem $X \leqq q$ where dem means demension in the sense of Štanko. Since $\operatorname{dim} \leqq$ dem, this is a stronger result than the classical $\operatorname{dim} X \leqq q$ [14, p. 104]. The third application deals with PL engulfing. The usual engulfing theorems [26, Chapter 4] are concerned with engulfing a polyhedron of dimension $r$. Using 6.9 we can show that the polyhedron can often be replaced by an arbitrary compact set of $\mathscr{H}^{r+1}$ measure zero.

6.13. Theorem. Let $M$ be a LIP n-manifold without boundary, let $E$ be closed in $M$ with $\mathscr{H}^{q}(E)=0$, and let $x_{0} \in M \backslash E$. Then the homomorphisms $\pi_{i}\left(M \backslash E, x_{0}\right) \rightarrow$ $\rightarrow \pi_{i}\left(M, x_{0}\right)$ induced by the inclusion are injective for $0 \leqq i \leqq n-q-1$ and surjective for $0 \leqq i \leqq n-q$.

Proof. Suppose first $0 \leqq i \leqq n-q$. Let $\alpha \in \pi_{i}\left(M, x_{0}\right)$. By 5.18, $\alpha$ has a LIP representative $f:\left(I^{i}, \partial I^{i}\right) \rightarrow\left(M, x_{0}\right)$. By 6.9, there is a LIP isotopy of $M$ which carries im $f$ off $E$ and keeps $x_{0}$ fixed. Hence $f$ is homotopic rel $\partial I^{i}$ to a map into $M \backslash E$.

Next assume $0 \leqq i \leqq n-q-1$. Let $f:\left(I^{i}, \partial I^{i}\right) \rightarrow\left(M \backslash E, x_{0}\right)$ be a map homotopic to the constant map $c$ rel $\partial I^{i}$ in $M$. We must show that $f \simeq c \operatorname{rel} \partial I^{i}$ in $M \backslash E$. By 5.18, we may assume that $f$ is LIP. Choose a homotopy $H:\left(I^{i} \times I, \partial I^{i} \times I\right) \rightarrow\left(M, x_{0}\right)$ from $f$ to $c$. By 5.18, we may again assume that $H$ is LIP. Now use 6.9 to isotope im $H$ off $E$ keeping $x_{0} \cup \operatorname{im} f$ fixed, and the theorem follows.

6.14. Remarks. The above result is true, of course, for PL and DIFF manifolds. For $i=0$ it means that no component of $M$ is contained in $E$ if $\mathscr{H}^{n}(E)=0$ and that each component of $M$ contains exactly one component of $M \backslash E$ if $\mathscr{H}^{n-1}(E)=0$. These are classical results, see [14, Theorem VII 3, p. 104 and Corollary 1, p. 48]. The theorem is also true for manifolds with boundary. A special case of 6.13 was proved in $[17,3.3]$.

6.15. Theorem. Let $X \subset R^{n}$ be compact with $\mathscr{H}^{q+1}(X)=0$. Then $\operatorname{dem} X \leqq q$.

Proof. We shall use the dual demension Dem of Štanko [28], see Edwards [9, Proposition $1.2\left(2^{\prime}\right)$ ]. Let $P$ be a closed polyhedron in $R^{n}$ with $\operatorname{dim} P=p \leqq n-$ $-q-1$, let $U$ be a neighborhood of $X \cap P$, and let $\varepsilon>0$. Then $P$ is countably $p$-rectifiable. By 6.10 , there is an $\varepsilon$-isotopy of $R^{n}$ supported by $U$ which carries $X$ off $P$. Hence $\operatorname{dem} X \leqq q$.

6.16. Theorem (Engulfing). Suppose that $M$ is an $r$-connected PL n-manifold without boundary with $r \leqq n-3$. If $X \subset M$ is compact and $\mathscr{H}^{r+1}(X)=0$, then $X$ is contained in a PL n-ball.

Proof. Choose a compact polyhedral neighborhood $Y$ of $X$ and a triangulation $K$ of $Y$ such that no simplex of $K$ meets both $X$ and $\partial Y$. Let $J_{1}$ be the $(n-r-1)$ - 
skeleton of $K$ and let $J_{2}=\{A \in K \mid A \cap X=\emptyset\}$. By 6.10, there is a PL homeomorphism $h: M \rightarrow M$ such that $\left|J_{1}\right| \cap h X=\emptyset$ and $h|(M \backslash Y) \cup| J_{2} \mid=\mathrm{id}$. Let $L$ be the subcomplex of the barycentric subdivision of $K$ consisting of those simplexes which do not meet $\left|J_{1}\right| \cup\left|J_{2}\right|$. Then $\operatorname{dim} L=r$. By the engulfing theorem of Stallings $[26$, p. 150], $|L|$ is contained in the interior of a PL ball $B \subset M$. Using the map described in 2.43 (not in 2.42!), we find a PL homeomorphism $g: M \rightarrow M$ which keeps $|L| \cup\left|J_{1}\right| \cup\left|J_{2}\right| \cup(M \backslash Y)$ fixed and maps int $B$ onto a set containing $h X$. Then $X$ is contained in the PL ball $h^{-1} g B$.

6.17. The above results deal with general position of sets. We have been less successful with the general position of maps. For example, we would like to show that a LIP map $M^{n} \rightarrow N^{2 n+1}$ can be LIP approximated by a LIP embedding, but we have not even been able to prove this in the euclidean case. However, we have established the following weaker result:

6.18. Theorem. Let $M$ be a compact LIP manifold with $\partial M=\emptyset$, and let $g: M \rightarrow R^{2 n+1}$ be a LIP map. Then for every $\varepsilon>0$ there is an injective LIP map $g_{1}: M \rightarrow R^{2 n+1}$ such that $d\left(g_{1}, g\right)<\varepsilon$ and lip $\left(g_{1}-g\right)<\varepsilon$.

Proof. Since the result is rather unsatisfactory, we only give a sketch. Let $E$ be the space of all LIP maps $M \rightarrow R^{2 n+1}$. Then $E$ is a Banach space with the norm $\|f\|=\sup _{x \in M}|f(x)|+\operatorname{lip} f$. For $\varepsilon>0$, the set $G_{\varepsilon}=\left\{f \in E \mid d\left(f^{-1}(y)\right)<\varepsilon\right.$ for all $\left.y \in R^{2 n+1}\right\}$ is open in $E$. By Baire's theorem, it suffices to show that $G_{\varepsilon}$ is dense. Let $f \in E$. Cover $M$ with interiors $U_{1}, \ldots, U_{k}$ of LIP balls such that $d\left(U_{i}\right)<\varepsilon$ for all $i$. Using 6.4 we can find a map $f_{1}$ such that $f_{1}(x)=f(x)$ for $x \in M \backslash U_{1}, f\left[M \backslash U_{1}\right] \cap$ $\cap f_{1} U_{1}=\emptyset$, and $\left\|f_{1}-f\right\|$ is small. Then modify similarly $f_{1}$ in $U_{2}$. After $k$ steps we obtain a map $f_{k}$ such that each fiber of $f_{k}$ is contained in some $U_{i}$. Thus $f_{k} \in G_{\varepsilon}$, and the theorem follows.

\section{Collaring and the Schönflies problem}

7.1. In this section we first prove a LIP version of an important result of Brown [5], which states that a locally collared set is collared. In particular, the boundary of a LIP manifold $M$ has a collar in $M$. Next we prove a Schönflies theorem in the LIP category. In particular, a PL $(n-1)$-sphere in $R^{n}$ always bounds a LIP ball.

7.2. Definitions. We let $I^{\prime}$ denote the interval $[0,1)$. Let $Y$ be a subset of a metric space $X$. A LIP collar of $Y$ in $X$ is a LIP embedding $c: Y \times I^{\prime} \rightarrow X$ such that $c(x, 0)=x$ for all $x \in Y$ and $\operatorname{im} c$ is an open neighborhood of $Y$ in $X$. A local LIP collar is a family $\left(U_{j}, c_{j}\right)_{j \in J}$ such that $\left(U_{j}\right)_{j \in J}$ is a cover of $Y, U_{j}$ is open in $Y$, and $c_{j}$ is a LIP collar of $U_{j}$ in $X$.

7.3. Lemma. Let $X$ be a metric space, let $Y \subset X$, let $c: Y \times I^{\prime} \rightarrow X$ be a map such that $c(x, 0)=x$ for all $x \in Y$, and let $\varepsilon: Y \rightarrow(0, \infty)$ be continuous. Then there 
is a LIP map $\delta: Y \rightarrow(0,1]$ such that $d(x, c(x, t))<\varepsilon(x)$ whenever $x \in Y$ and $0 \leqq t<$ $<\delta(x)$.

Proof. For $x \in Y$ set $g(x)=\sup \{t \mid c[x \times[0, t]] \subset B(x, \varepsilon(x))\}$. It is easy to see that $g$ is lower semicontinuous. By 5.4, there is a LIP map $\delta: Y \rightarrow R^{1}$ such that $0<\delta(x)<g(x)$ for all $x$.

7.4. Theorem. Let $Y$ be a subset of a metric space $X$. If $Y$ has a local LIP collar in $X$, then $Y$ has a LIP collar in $X$.

Proof. Let $\mathscr{U}$ be the family of all sets $U$ open in $Y$ such that $U$ has a LIP collar in $X$. Then $\mathscr{U}$ is a cover of $Y$. It suffices to show that $\mathscr{U}$ satisfies the conditions of 5.8. The condition (1) is clear. Next it is easy to verify that Brown's proof for the TOP case of (3) [26, Lemma 1.7.1, p. 35] yields a LIP collar if all given collars are LIP. We shall prove (2) using an idea of Connelly [6]. However, since we do not assume that $Y$ is closed in $X$, an additional argument is needed.

Assume that $U_{1}, U_{2} \in \mathscr{U}$, and set $U=U_{1} \cup U_{2}$. Let $c_{i}: U_{i} \times I^{\prime} \rightarrow X$ be a LIP collar of $U_{i}, i=1,2$. Applying 7.3 we find LIP maps $\delta_{i}: U_{i} \rightarrow(0,1]$ such that $d\left(x, c_{i}\left(x, \delta_{i}(x) t\right)\right)<d\left(x, U \backslash U_{i}\right)$ for all $(x, t) \in U_{i} \times I^{\prime}, \quad i=1,2$. Thus $d\left(c_{i}\left(x, \delta_{i}(x) t\right), U \backslash U_{i}\right)>0$ for all $(x, t) \in U_{i} \times I^{\prime}$. Replacing $c_{i}$ by the LIP collar $(x, t) \mapsto c_{i}\left(x, \delta_{i}(x) t\right)$ we may therefore assume that $U$ is closed in $N=\operatorname{im} c_{1}$ vim $c_{2}$. Moreover, we may assume that $c_{i}^{-1} U=U_{i} \times 0$. From now on, all closures will be taken in $N$. Choose a LIP partition of unity $\left(\varphi_{1}, \varphi_{2}\right)$ on $U$ such that $\operatorname{spt} \varphi_{i}=$ $=A_{i} \subset U_{i}$. Then choose an open neighborhood $V_{i}$ of $A_{i}$ in $N$ such that $\bar{V}_{i} \subset \operatorname{im} c_{i}$. Setting $U_{i}^{\prime}=V_{i} \cap Y$ we have $U=U_{1}^{\prime} \cup U_{2}^{\prime}$. By 7.3, there are LIP maps $\delta_{i}: U_{i}^{\prime} \rightarrow(0,1]$ such that $d\left(x, c_{i}\left(x, \delta_{i}(x) t\right)\right)<d\left(x, N \backslash V_{i}\right)$ for all $(x, t) \in U_{i}^{\prime} \times I^{\prime}$. Setting $B_{i}=$ $=\left\{c_{i}\left(x, \delta_{i}(x) t\right) \mid x \in A_{i}, 0 \leqq t \leqq 1 / 2\right\}$ we thus have $B_{i} \subset V_{i}$. Since $B_{i}$ is closed in im $c_{i}$ and $\bar{B}_{i} \subset \bar{V}_{i} \subset \operatorname{im} c_{i}, B_{i}$ is closed in $N$. Replacing $U_{i}$ by $U_{i}^{\prime}, c_{i}$ by $c_{i}^{\prime}(x, t)=\left(x, \delta_{i}(x) t\right)$, and $N$ by im $c_{1}^{\prime} \cup \operatorname{im} c_{2}^{\prime}$, we may therefore assume that $c_{i}\left[A_{i} \times[0,1 / 2]\right]$ is closed in $N$ for $i=1,2$.

Set $M=N \cup U \times[-1,0]$, where $(x, 0)$ is identified with $x$. We shall construct a lipeomorphism $g: N \rightarrow M$ such that $g(x)=(x,-1)$ for $x \in U$. Then $(x, t) \mapsto$ $\mapsto g^{-1}(x, t-1)$ will give a LIP collar of $U$ in $N$, and hence in $X$.

Define $h_{i}: U_{i} \times[-1,1) \rightarrow M$ by $h_{i}(x)=c_{i}(x)$ for $x \in U_{i} \times I^{\prime}$ and by $h_{i}(x)=x$ otherwise. It is easy to see, for example by 2.22 , that $h_{i}$ is a LIP embedding. Let $f_{1}: U_{1} \times I^{\prime} \rightarrow U_{1} \times[-1,1)$ be defined by $f_{1}(x, t)=\left(x, r_{x}(t)\right)$ where $r_{x}(t)=t$ for $t \in[1 / 2,1)$ and $r_{x}$ maps $[0,1 / 2]$ affinely onto $\left[-\varphi_{1}(x), 1 / 2\right]$. From 2.40 it follows that $f_{1}$ is a LIP embedding. Next define $g_{1}: N \rightarrow M$ by $g_{1}(x)=h_{1}\left(f_{1}\left(h_{1}^{-1}(x)\right)\right)$ for $x \in \operatorname{im} c_{1}$ and by $g_{1}(x)=x$ otherwise. Since $c_{1}\left[A_{1} \times[0,1 / 2]\right]$ is closed in $N, g_{1}$ is a LIP embedding. Moreover, $h_{2}^{-1} g_{1} N=\left\{(x, t) \mid x \in U_{2}, \quad-\varphi_{1}(x) \leqq t<1\right\}$. Let $f_{2}: h_{2}^{-1} g_{1} N \rightarrow U_{2} \times[-1,1)$ be defined by $f_{2}(x, t)=\left(x, s_{x}(t)\right)$ where $s_{x}(t)=t$ for $t \in[1 / 2,1)$ and $s_{x}$ maps $\left[-\varphi_{1}(x), 1 / 2\right]$ affinely onto $[-1,1 / 2]$. By $2.40, f_{2}$ is a lipeomorphism. Define $g_{2}: g_{1} N \rightarrow M$ by $g_{2}(x)=h_{2}\left(f_{2}\left(h_{2}^{-1}(x)\right)\right)$ for $x \in \operatorname{im} h_{2} \cap g_{1} N$ 
and by $g_{2}(x)=x$ otherwise. Since $h_{2}\left[A_{2} \times[-1,1 / 2]\right]$ is closed in $M, g_{2}$ is a lipeomorphism. Then $g=g_{2} g_{1}: N \rightarrow M$ is the sought-for lipeomorphism.

7.5. Corollary. If $M$ is a LIP manifold, then $\partial M$ has a LIP collar in $M$.

7.6. Schönflies problem. Let $f: S^{n-1} \rightarrow S^{n}$ be a topological embedding. If $n=2$, then $f$ can be extended to a homeomorphism $S^{n} \rightarrow S^{n}$, according to the classical Schönflies theorem. Because of the wild embeddings, the result is not true for $n \geqq 3$. However, if $f$ can be extended to an embedding $g$ of an annulus $\bar{B}^{n} \backslash B^{n}(a), 0<a<1$, then $f$ can be extended to a homeomorphism of $\bar{B}^{n}$ onto $\bar{D}$ where $D$ is the component of $S^{n} \backslash f S^{n-1}$ containing $g\left[B^{n} \backslash B^{n}(a)\right]$. This result is due to Brown [3] and also to Mazur [18] and Morse [22]. Similarly, we may consider the CAT Schönflies problem, where CAT is one of the categories DIFF, PL, LIP, TOP: Suppose that $B$ is a CAT $n$-ball, $S$ a CAT $n$-sphere, $f$ a CAT embedding of a neighborhood of $\partial B$ in $B$ into $S$. Does $f \mid \partial B$ have an extension to a CAT embedding of $B$ into $S$ ? Note that in this case, $f \partial B$ has a CAT collar in $\bar{D}$ where $D$ is as above. In other words, $\bar{D}$ is a CAT manifold with boundary $f \partial B$. Stated in this form, the answer is known to be negative for $\mathrm{CAT}=\mathrm{DIFF}$, since there are diffeomorphisms $S^{n-1} \rightarrow S^{n-1}$ which cannot be extended to a diffeomorphism of $\bar{B}^{n}$. In the PL case, the answer is positive for $n \neq 4$ and unknown for $n=4$. We shall show that the answer is positive for CAT=LIP. Without any collaring condition, a LIP $(n-1)$-sphere in $S^{n}$ need not even bound a topological ball. A counterexample can be constructed with the aid of a fattened Fox-Artin arc, see 3.10.

A quasiconformal Schönflies theorem was proved by Gehring [11], who used an explicit version of the Mazur-Morse method. His proof needs only slight changes to yield the LIP theorem. To avoid repetition, we refer to Gehring's proof as given in [29, Section 41] and give only the modifications needed in the LIP case.

7.7. Theorem. Suppose that $0<a<1$ and that $f$ is $a$ LIP embedding of the annulus $E=\bar{B}^{n} \backslash B^{n}(a)$ into $S^{n}$. Then $f \mid S^{n-1}$ can be extended to a LIP embedding $f^{*}: \bar{B}^{n} \rightarrow S^{n}$.

Proof. We may assume $n \geqq 2$. We work in the compactified space $\bar{R}^{n}=R^{n} \cup \infty$. With the spherical metric $q\left[29\right.$, p. 37], it is a metric space lipeomorphic to $S^{n}$. Moreover, the identity map is a lipeomorphism of $\left(R^{n}, q\right)$ onto $R^{n}$ with the euclidean metric. All complements and closures are taken in $\bar{R}^{n}$.

Step 1. (See $[29,41.1]$.) Suppose that (1) $D_{1}, D_{2}$ are domains such that $\bar{D}_{1} \cap \bar{D}_{2}=\emptyset$ and $\bar{D}_{1} \cup \bar{D}_{2} \subset B^{n}$, (2) $B_{1}$ and $B_{2}$ are open round balls such that $\bar{B}_{1} \cap$ $\cap \bar{B}_{2}=\emptyset$ and $\bar{B}_{1} \cup \bar{B}_{2} \subset B^{n}$, (3) $f$ is a lipeomorphism of $\mathbf{C}\left(D_{1} \cup D_{2}\right)$ onto $\mathbf{C}\left(B_{1} \cup B_{2}\right)$ such that $f \partial D_{i}=\partial B_{i}$, (4) $f(x)=x$ in a neighborhood of $\mathbf{C} B^{n}$. Then there exists a lipeomorphism $f^{*}: \mathbf{C} D_{2} \rightarrow \mathbf{C} B_{2}$ such that $f^{*}\left|\partial D_{2}=f\right| \partial D_{2}$. All LIP properties are taken in the spherical metric. 
The construction of $f^{*}$ is exactly as in [29]. Only the LIP property of $f^{*}$ at $\infty$ needs a separate argument.

The set $A=\mathbf{C} B^{n} \backslash \infty$ is ( $\left.\pi / 2\right)$-quasiconvex in the euclidean metric. Moreover, $A$ is a locally finite union of closed sets $A_{i}$ such that each $f^{*} \mid A_{i}$ is a composite map of translations, $f, f^{-1}$, and a single affine map, common to all $i$. By $2.35, f^{*} \mid A$ is $L$-Lipschitz for some $L$ in the euclidean metric. Since $\left|f^{*}(x)-x\right|<5$ for all finite $x$, $\left|f^{*}(x)\right| \geqq|x| / 2$ for $|x| \geqq 10$. Therefore, for all $x, y$ in $\mathbf{C} B^{n}(10) \backslash \infty$, we obtain

$$
\begin{aligned}
q\left(f^{*}(x), f^{*}(y)\right) & =\left|f^{*}(x)-f^{*}(y)\right|\left(1+\left|f^{*}(x)\right|^{2}\right)^{-1 / 2}\left(1+\left|f^{*}(y)\right|^{2}\right)^{-1 / 2} \\
& \leqq 4 L q(x, y) .
\end{aligned}
$$

Hence $f^{*}$ is LIP at $\infty$. A similar argument shows that $\left(f^{*}\right)^{-1}$ is LIP at $\infty$, and Step 1 is proved.

Step 2. (See $[29,41.2]$.) In addition to (1), (2), and (3) of Step 1, suppose that (4') $0 \in D_{2}$, (5') $\mathbf{C} f \mathbf{C} \bar{B}^{n} \subset B^{n}$. Then there exists a lipeomorphism $f^{*}: \mathbf{C} D_{2} \rightarrow \mathbf{C} B_{2}$ such that $f^{*}\left|\partial D_{2}=f\right| \partial D_{2}$.

The proof of Step 2 in [29] is also valid in our case except that we must replace the map $g$ of [29] by the following map $g: \bar{R}^{n} \rightarrow \bar{R}^{n}$ (which could be used in the qc case as well): $g(0)=0, g(\infty)=\infty$, and $g(x)=\varphi(|x|)|x|^{-1} x$ for $x \neq 0$, $\infty$, where $\varphi: R_{+}^{1} \rightarrow R_{+}^{1}$ is the PL homeomorphism which maps $[0, a]$ linearly onto $[0, b],[a, 1]$ affinely onto $[b, 1]$ and is the identity on $[1, \infty)$. Then $g$ is a lipeomorphism.

Step 3. The proof of $[29,41.3]$ can be directly translated to the LIP case. Since Möbius transformations are diffeomorphisms of $S^{n}$, they are lipeomorphisms in the spherical metric of $\bar{R}^{n}$. This completes the proof of 7.7.

7.8. Theorem. Let $S$ be a LIP $n$-sphere, and let $S_{1}$ be a locally LIP flat LIP $(n-1)$-sphere in $S$. Then $\left(S, S_{1}\right)$ is lipeomorphic to the standard pair $\left(S^{n}, S^{n-1}\right)$.

Proof. Choose a lipeomorphism $h: S^{n-1} \rightarrow S_{1}$. Let $D_{1}, D_{2}$ be the components of $S \backslash S_{1}$. Since $S_{1}$ is locally LIP flat, it has a local LIP collar in $\bar{D}_{1}$. By 7.4 , it has a LIP collar $c: S_{1} \times I^{\prime} \rightarrow \bar{D}_{1}$ in $\bar{D}_{1}$. Then $h$ can be extended to a LIP embedding $f: \bar{B}^{n} \backslash 0 \rightarrow \bar{D}_{1}$ by $f(x)=c(h(x /|x|), 1-|x|)$. By $7.7, h$ can be extended to a lipeomorphism $g_{1}: \bar{B}^{n} \rightarrow \bar{D}_{1}$. Similarly we find an extension of $h$ to a lipeomorphism $g_{2}: \bar{R}^{n} \backslash B^{n} \rightarrow \bar{D}_{2}$. Then $g_{1} \cup g_{2}$ is a lipeomorphism of $\left(\bar{R}^{n}, S^{n-1}\right)$ onto $\left(S, S_{1}\right)$. Here $\bar{R}^{n}=R^{n} \cup \infty$ with the spherical metric.

7.9. Theorem. Let $M$ be a compact LIP n-manifold which is the union of two open LIP $n$-balls. Then $M$ is a LIP $n$-sphere.

Proof. See the TOP case [26, Theorem 1.8.4, p. 49].

7.10. Theorem. Let $S$ be a PL n-sphere and let $S_{1} \subset S$ be a PL (n-1)-sphere. Then $\left(S, S_{1}\right)$ is lipeomorphic to the standard pair $\left(S^{n}, S^{n-1}\right)$. In particular, the closure of each component of $S \backslash S_{1}$ is a LIP $n$-ball. 
Proof. The theorem is obvious for $n=1$. Proceeding inductively, we assume that the theorem is true for $n \leqq p$, and suppose $n=p+1$. By 7.8, it suffices to show that $S_{1}$ is locally LIP flat in $S$. Let $x \in S_{1}$, and choose a triangulation $(K, L)$ of $\left(S, S_{1}\right)$ such that $x$ is a vertex. By induction, the PL sphere pair $(|1 \mathrm{k}(x, K)|,|1 \mathrm{k}(x, L)|)$ is lipeomorphic to $\left(S^{p}, S^{p-1}\right)$. The cone construction yields a lipeomorphism of $(\mid$ st $(x, K)|$,$| st (x, L) \mid)$ onto $\left(\bar{B}^{p+1}, \bar{B}^{p}\right)$. Hence $S_{1}$ is locally LIP flat in $S$.

\section{LIP structures of $R^{n}, S^{n}$, and $I^{n}$}

8.1. The aim of this section is to prove the Lipvermutung (LIP Hauptvermutung) for the manifolds $R^{n}$ and $S^{n}$ for $n \neq 4$, and for $I^{n}$ for $n \neq 4,5$. By this we mean that if a LIP manifold is homeomorphic to one of these manifolds, it is lipeomorphic to it. A LIP version of the Poincaré conjecture follows then directly from the corresponding TOP result, proved by Newman [24]. In fact, this section is a slightly enlarged LIP version of Newman's paper. We begin by the LIP version of Newman's engulfing theorem [24, Theorem 5]. A subset $X$ of a LIP $n$-manifold $M$ is called LIP $p$-dominated if for every $x \in X$ there is a neighborhood $N$ of $x$ and a lipeomorphism $f: N \rightarrow I^{n}$ such that $f[N \cap X]$ is contained in a polyhedron of dimension at most $p$.

8.2. Theorem (Engulfing). Let $M$ be a LIP n-manifold without boundary, and let $X \subset M$ be closed and LIP $p$-dominated with $p \leqq n-3$. Let $V$ be an open set in $M$ such that $(M, V)$ is p-connected and $X \backslash V$ is compact. Then there is a lipeomorphism $h: M \rightarrow M$ such that $X \subset h V$ and $h=\mathrm{id}$ outside a compact set.

Proof. The theorem is proved by rewriting Newman's proof in the LIP category. It is only necessary to be sure that all maps occurring in the proof can be made LIP. The previous sections of our paper give all the tools needed for this. Since the proof is long, we must leave the details to the reader. However, we shall give some hints for this translation work together with some remarks on Newman's proof [24, Sections 1-15, pp. 555-569] concerning omission of certain unneeded hypotheses. and correction of some slight inaccuracies.

Lemma 1. To obtain a LIP map $\varphi$, apply 5.4.

Lemma 2. Assume that $X$ is a LIP manifold and $F$ compact. In the proof, set $f_{t}=(1-t) f_{0}+t f_{1}$ and replace $f_{1+}$ by $f_{b}$ and $\varphi^{\prime}$ by $f_{a}$ for suitable $0<a<1<b$. A computation shows that $h \mid \pi\left(f_{0}, f_{b}\right)$ is LIP. By $2.36, h$ is LIP. Similarly $h^{-1}$ is LIP. In the definition of engulfing, the isotopy condition (2) can be omitted.

Theorem 1. Assume that $Y$ and $Z$ are LIP manifolds, $F$ is compact, and $g_{1}$ is LIP. In case 1 of the proof, assume that $g_{0}$ is also LIP. The set $V$ is chosen to be $A \times \operatorname{int}_{\mathrm{Z}} F$ where $A$ is a relatively compact neighborhood of $o$. Defining $\varphi(y, z)=$ $=\left(d(y, o) g_{0}(z)+d(y, \partial A) g_{1}(z)\right) /(d(y, o)+d(y, \partial A))$ yields a LIP engulfing $h$. The theorem is only needed with (4.3) replaced by $\left(4.3^{\prime}\right)$. 
Theorem 3. Assume that $L, K$ are compact and $f$ is LIP. Since $f^{\prime}$ is constructed by a coning process, it follows from our $\S 2$ that $f^{\prime}$ is LIP.

Theorem 4. Assume that $L, K$ are compact and $f$ is LIP. Newman's map $\varphi$ seems to be a standard mistake of the second kind (see 2.43), but this is easily corrected.

Theorem 6. Assume that $H, L, \Gamma$ are compact, $M$ a LIP manifold, $X$ LIP $p$-dominated, and $f$ LIP. Replace the condition $\operatorname{dim} \Gamma \leqq p$ by the following one: $\operatorname{dim} \Gamma \leqq n$ and $\operatorname{dim} H \leqq p$. Indeed, the theorem will be applied on p. 569 with the substitution $\Gamma \mapsto G^{*}$ where $\operatorname{dim} G^{*}$ may be $n-2$. Claim that $g$ is LIP and $h$ is a lipeomorphism.

Section 11. Replace the additional condition of $B(q)$ by the following one: $H \backslash f^{-1} V$ has a neighborhood in $H$ of dimension $\leqq q$. This helps in the proof of $C(q, 1) \Rightarrow C(q, m)$ and allows us to change (11.2) into the form: int $\sigma^{q}$ is a neighborhood of $H \backslash f^{-1} V$ in $H$. This in turn will imply in Section 12 that $\sigma^{q}$ is a principal simplex of $H$.

Lemma 6. In the proof, choose $\Gamma_{0}$ so that $\operatorname{dim} \overline{H \backslash P} \leqq q$.

Section 12. By 5.18, the extended map $f$ can be chosen to be LIP.

Lemma 7. Assume that $G, L, K$ are compact, $\operatorname{dim} G \leqq n, \operatorname{dim} K \leqq p$, and $f$ is LIP. In the proof, we could not see why the map $g_{i+1}$ satisfies (13.3): $g_{i+1} \mid L \cup G^{\alpha}=$ $=f^{\prime} \mid L \cup G^{\alpha}$, although obviously $g_{i+1}(x)=f^{\prime}(x)$ for $x \in L \cup\left(G^{\alpha} \cap D\right)$. We obtained $g_{i+1}$ by applying 5.17 with the substitution $X \mapsto G, Y \mapsto L \cup G^{\alpha} \cup D, f_{\mapsto} \mapsto f^{\prime}, g \mapsto\left(f^{\prime} \mid L \cup G^{\alpha}\right) \cup$ $\cup\left(g^{\prime} \mid L \cup D\right)$. Moreover, when defining the polyhedron $G^{*}$, one should identify points in $L \cup\left(D \cap G^{\alpha}\right)$ with the same $g_{i}$-image, not only in $D \cap G^{\alpha}$, since otherwise $f^{*} \mid L^{*}$ is not necessarily an embedding. When applying $B(q-1)$ on p. 569 , the new condition of $B(q-1)$ is satisfied, since $W=D^{*} \backslash p\left[\left.K \cup\right|_{i} Q_{1}^{q} \mid\right]$ is a neighborhood of $D^{*} \backslash f^{*-1} h_{i} V$ in $D^{*}$ and $W \subset p P$.

The rest of the proof is essentially unchanged.

8.3. Theorem. Let $X$ be a metric space which is the union of open sets $V_{1} \subset$ $\subset V_{2} \subset \ldots$ such that every $V_{i}$ is lipeomorphic to $R^{n}$. Then $X$ is lipeomorphic to $R^{n}$.

Proof. The corresponding TOP result is due to Brown [4]. It is easy to check that all maps in Brown's paper can be made LIP.

8.4. Theorem. Let $M$ be a LIP manifold homeomorphic to $R^{n}, n \neq 4$. Then $M$ is lipeomorphic to $R^{n}$.

Proof. The case $n=1$ follows from 3.11. For $n \geqq 2$, Theorem 8.3 implies that it is sufficient to show that each compact set $A \subset M$ is contained in an open LIP ball. We divide the rest of the proof into two cases: $2 \leqq n \leqq 3$ and $n \geqq 5$.

Let $n=2$ or 3 , and let $A \subset M$ be compact. Choose a homeomorphism $h: M \rightarrow R^{n}$ 
and a closed $n$-cube $Q$ containing $h A$. Divide $Q$ into congruent closed cubes $Q_{1}, \ldots, Q_{k}$ so that for each $i, P_{i}=Q_{1} \cup \ldots \cup Q_{i}$ is a PL $n$-ball, $P_{i} \cap Q_{i+1}$ is a PL $(n-1)$-ball in $\partial Q_{i+1}$, and $Q_{i}^{\prime}=h^{-1} Q_{i}$ is contained in an open LIP $n$-ball $B_{i} \subset M$. It suffices to show that $P_{i}^{\prime}=h^{-1} P_{i}$ is contained in an open LIP ball for all $i=1, \ldots, k$. This is clearly true for $i=1$. Proceeding inductively, assume that $P_{i}^{\prime}$ is contained in an open LIP ball $B$.

We can obviously choose an open neighborhood $U$ of $Q_{i+1}^{\prime}$ in $B_{i+1}$ and a homeomorphism $f$ of $U$ onto an open set $V \subset R^{n}$ such that $f Q_{i+1}^{\prime}=I^{n-1} \times I$ and $f\left[U \cap P_{i}^{\prime}\right] \subset$ $\subset\left\{x \in R^{n} \mid x_{n} \leqq 0\right\}$. Choose $\varepsilon \in(0,1)$ such that $(1+\varepsilon)\left(I^{n-1} \times I\right) \subset V$ and $(1+\varepsilon) I^{n-1} \times$ $\times[0, \varepsilon] \subset f[U \cap B]$. Let $S$ be the PL $(n-1)$-sphere $\partial\left((1+\varepsilon) I^{n-1} \times[\varepsilon, 1+\varepsilon]\right)$, and let $S_{1}$ be the PL $(n-2)$-sphere $\partial\left((1+\varepsilon) I^{n-1} \times \varepsilon\right)$. Choose a lipeomorphism $g$ of $U$ onto an open set $W \subset R^{n}$. Then $\varphi=g f^{-1}: V \rightarrow W$ is a homeomorphism. For every $\delta>0$, there is a homeomorphism $u$ of $\varphi S$ onto a PL $(n-1)$-sphere $E$ such that $d(u, \mathrm{id})<\delta$. For $n=2$ this is elementary, and for $n=3$ this follows from the approximation theorem of Bing [1, Theorem 1]. The same argument in the dimension $n-1$ yields a homeomorphism $v$ of $u \varphi S_{1}$ onto a PL $(n-2)$-sphere $F \subset E$ such that $d(v, \mathrm{id})<\delta$. Let $D_{1}, D_{2}$ be the components of $E \backslash F$, and let $D$ be the bounded component of $R^{n} \backslash E$. Choosing $\delta$ small enough, we may assume that $g\left[Q_{i+1}^{\prime} \backslash B\right] \subset D$, $\bar{D} \subset W, \bar{D}_{1} \subset g[B \cap U]$, and $E \cap g Q_{i+1}^{\prime} \subset D_{1}$.

By 7.10 or by the PL Schönflies theorem, $\bar{D}$ is a LIP ball. Choose a lipeomorphism $q: \bar{D} \rightarrow I^{n}$. Also by $7.10,(E, F)$ is lipeomorphic to $\left(\partial I^{n}, \partial I^{n-1}\right)$. Using the cone construction, we may therefore assume that $q D_{2}=\partial I^{n} \cap R_{+}^{n}$. It is easy to construct LIP maps $\alpha, \beta: I^{n-1} \rightarrow(0,1)$ such that $\alpha\left|\partial I^{n-1}=\beta\right| \partial I^{n-1}=0,\{(x, t) \mid-1 \leqq t \leqq \alpha(x)\} \subset$ $\subset q[\bar{D} \cap g[B \cap U]]$, and $q\left[\bar{D} \cap g Q_{i+1}^{\prime}\right] \subset\{(x, t) \mid-1 \leqq t<\beta(x)\}$. Applying 2.40 we find a lipeomorphism $r: I^{n} \rightarrow I^{n}$ such that $r \mid \partial I^{n}=$ id and $r(x, \alpha(x))=(x, \beta(x))$ for all $x \in I^{n-1}$. Define $w: M \rightarrow M$ by $w\left|g^{-1} D=g^{-1} q^{-1} r q g\right| g^{-1} D$ and by $w \mid M \backslash g^{-1} D=$ id. Then $w$ is a lipeomorphism, and $w B$ is an open LIP ball containing $P_{i+1}^{\prime}$. The case $2 \leqq n \leqq 3$ is proved.

Suppose that $n \geqq 5$. Assume that $A \subset M$ is compact. Using the proof of Newman [24, Theorem 7, p. 570], attributed by Newman to Connell, we can first show that $A$ can be covered with two open LIP balls. The proof makes use of 2.42 and 8.2. Then a slight modification of this proof shows that $A$ can be covered with a single open LIP ball. We shall give the latter proof in detail.

We may assume that $A$ is a locally flat TOP $n$-ball. Choose LIP balls $B_{1}, B_{2}$ with $A \subset$ int $B_{1} \cup$ int $B_{2}$. Using lipeomorphisms $B_{i} \rightarrow I^{n}$ we introduce PL structures on $B_{i}, i=1,2$. Choose smaller concentric "cubes" $C_{i} \subset \operatorname{int} B_{i}, D_{i} \subset \operatorname{int} C_{i}, i=1,2$, such that $A \subset \operatorname{int} D_{1}$ vint $D_{2}$. Choose a triangulation $K$ of $C_{2}$ such that a subcomplex triangulates $D_{2}$ and no simplex meets both $\partial D_{1}$ and $\partial C_{1}$. Set $M_{1}=$ $=M \backslash C_{1}, V_{1}=$ int $B_{1} \backslash C_{1}, X_{1}=\left|K^{2}\right| \backslash C_{1}$. Since $M_{1}$ and $V_{1}$ are 2-connected, it follows from 8.2 that there is a lipeomorphism $h_{1}: M_{1} \rightarrow M_{1}$ such that $h_{1} V_{1} \supset X_{1}$ and $h_{1}=$ id near $\partial M_{1}$. We extend $h_{1}$ by identity to a lipeomorphism $h_{1}: M \rightarrow M$. Then $U_{1}=h_{1}$ int $B_{1}$ is an open LIP ball and $\left|K^{2}\right| \cup C_{1} \subset U_{1}$. 
Next choose a derived subdivision of $K$, and let $L$ be the dual skeleton of $K^{2}$. Choose a TOP ball $A_{1}$ such that $A \subset A_{1} \subset D_{1} \cup D_{2}$ and $A_{1} \backslash$ int $A$ is homeomorphic to $S^{n-1} \times I$. Set $M_{2}=\operatorname{int} A_{1}, V_{2}=\operatorname{int} A_{1} \backslash A, X_{2}=|L| \cap \operatorname{int} A_{1}$. Since $M_{2}$ and $V_{2}$ are $(n-3)$-connected, it follows from 8.2 that there is a lipeomorphism $h_{2}: M_{2} \rightarrow M_{2}$ such that $h_{2} V_{2} \supset X_{2}$ and $h_{2}=$ id near $\partial M_{2}$. We extend $h_{2}$ by identity to a lipeomorphism $h_{2}: M \rightarrow M$. Then $|L| \cup\left(M \backslash A_{1}\right) \subset h_{2}[M \backslash A]=U_{2}$.

We have now the situation described at the end of 2.42. Hence there is a stretching lipeomorphism $h: C_{2} \rightarrow C_{2}$ such that $D_{2} \subset h\left[U_{1} \cap C_{2}\right] \cup\left(U_{2} \cap C_{2}\right)$ and $h \mid \partial C_{2}=\mathrm{id}$. We extend $h$ by identity to a lipeomorphism $h: M \rightarrow M$. Since $h$ maps simplexes of $K$ onto themselves, $D_{1} \subset h C_{1} \subset h U_{1}$. Thus $M=h U_{1} \cup U_{2}$, and $A$ is contained in the open LIP ball $h_{2}^{-1} h U_{1}$.

8.5. Theorem. Let $M$ be a LIP manifold homeomorphic to $S^{n}, n \neq 4$. Then $M$ is lipeomorphic to $S^{n}$.

Proof. Fix a point $x \in M$. Choose a LIP ball neighborhood $B_{1}$ of $x$. By 8.4, $S^{n} \backslash x$ is lipeomorphic to $R^{n}$. Hence there is a LIP ball $B_{2} \subset S^{n} \backslash x$ with $\partial B_{1} \subset$ int $B_{2}$. The theorem follows from 7.9.

8.6. Theorem. Let $M$ be a LIP manifold homeomorphic to $I^{n}, n \neq 4,5$. Then $M$ is lipeomorphic to $I_{:}^{n}$.

Proof. By 3.13, the double $D M$ of $M$ is a LIP manifold containing $M$ as a locally LIP flat submanifold. By $8.5, D M$ is a LIP $n$-sphere and $\partial M$ is a LIP $(n-1)$ sphere. By $7.8, M$ is a LIP $n$-ball.

\section{Open problems}

9.1. Elementary problems. (1) Can every LIP $n$-manifold be LIP embedded into $R^{2 n+1}$ ?

(2) The LIP annulus conjecture for $n=2$ : Let $S_{1}$ and $S_{2}$ be disjoint locally LIP flat LIP 1-spheres in a LIP 2-sphere $S$, and let $D$ be the domain whose boundary is $S_{1} \cup S_{2}$. Is $\bar{D}$ lipeomorphic to $S^{1} \times I$ ?

(3) Is a LIP arc in $R^{2}$ always locally LIP flat?

(4) More generally, is a locally TOP flat LIP arc in $R^{n}$ always locally LIP flat?

(5) Does a locally quasiconvex metric space have a basis consisting of quasiconvex sets?

9.2. Advanced problems. (1) The LIP annulus conjecture for $n \geqq 3$.

(2) Does every TOP manifold have a LIP structure?

(3) Lipvermutung: If two LIP manifolds are homeomorphic, are they lipeomorphic? 
(4) If two PL manifolds are lipeomorphic, are they PL homeomorphic?

9.3. Remarks. By Siebenmann [27, 2.1, p. 137], there exists a LIP manifold which has no PL structure. The answers to the questions (3) and (4) of 9.2 cannot be both positive, since they would yield the PL Hauptvermutung.

\section{References}

[1] Bing, R. H.: Approximating surfaces with polyhedral ones. - Ann. of Math. (2) 65, 1957, 456-483.

[2] Bing, R. H.: A simple closed curve that pierces no disk. - J. Math. Pures Appl. 35, 1956, $337-343$.

[3] Brown, M.: A proof of the generalized Schoenflies theorem. - Bull. Amer. Math. Soc. 66, 1960, 74--76.

[4] Brown, M.: The monotone union of open $n$-cells is an open $n$-cell. - Proc. Amer. Math. Soc. $12,1961,812-814$.

[5] Brown, M.: Locally flat imbeddings of topological manifolds. - Ann. of Math. (2) 75, 1962, $331-341$.

[6] Connelly, R.: A new proof of Brown's collaring theorem. - Proc. Amer. Math. Soc. 27, 1971, 180-182.

[7] DE RhAM, G.: Variétés différentiables. - Hermann et $C^{\text {ie }}$, Paris, 1955.

[8] DugundJ, J.: Topology. - Allyn and Bacon, Inc. Boston, 1966.

[9] EDwards, R.: Demension theory. - Geometric topology. - Lecture Notes in Mathematics 438 , Springer-Verlag, Berlin-Heidelberg-New York, 1975, 195-211.

[10] FedereR, H.: Geometric measure theory. - Springer-Verlag, Berlin-Heidelberg-New York, 1969.

[11] Gehring, F. W.: Extension theorems for quasiconformal mappings in $n$-space. - J. Analyse Math. 19, 1967, 149-169.

[12] Gehring, F. W.: Extension theorems for quasiconformal mappings in $n$-space. - Proc. Int. Congress Math. Moscow, 1968, 313-318.

[13] HANneR, O.: Some theorems on absolute neighborhood retracts. - Ark. Mat. 1, 1951, 389408.

[14] Hurewicz, W., and H. Wallman: Dimension theory. - Princeton University Press, Princeton, N. J., 1941.

[15] KatĚtov, M.: On quasi-metric properties (Russian). - Studia Math. (Ser. Specjalna) Zeszyt 1, 1963, 57-68.

[16] KLeE, V. L.: On a theorem of Béla Sz.-Nagy. - Amer. Math. Monthly 60, 1953, 618 -619.

[17] Martio, O., S. RickmAn, and J. VÄISÄLä: Topological and metric properties of quasiregular mappings. - Ann. Acad. Sci. Fenn. Ser. A I 488, 1971, 1-31.

[18] Mazur, B.: On imbeddings of spheres. - Bull. Amer. Math. Soc. 65, 1959, 59-65.

[19] McShane, E. J.: Extension of range of functions. - Ibid. 40, 1934, 837-842.

[20] Michael, E.: Local properties of topological spaces. - Duke Math. J. 21, 1954, 163-171.

[21] Milnor, J.: Differential topology. - Mimeographed notes, Princeton University, Princeton, N. J., 1958.

[22] Morse, M.: A reduction of the Schoenflies extension problem. - Bull. Amer. Math. Soc. 66, 1960, 113-115.

[23] Munkres, J.: Elementary differential topology. - Annals of Mathematics Studies, No. 54, Princeton University Press, Princeton, N. J., 1963. 
[24] Newman, M. H. A.: The engulfing theorem for topological manifolds. - Ann. of Math. (2) $84,1966,555-571$.

[25] Rourke, C. P., and B. J. Sanderson: Introduction to piecewise-linear topology. - SpringerVerlag, Berlin-Heidelberg-New York, 1972.

[26] Rushing, T. B.: Topological embeddings. - Academic Press, New York-London, 1973.

[27] Siebenmann, L. C.: Topological manifolds. - Proc. Int. Congress Math. Nice 2, 1971, 133163.

[28] S̆TAN'Ko, M. A.: Imbedding of compacta in euclidean space. - Mat. Sb. (N. S.), 83 (125), 1970, 234-255 (Russian).

[29] V̈̈ISÄL ̈̈, J.: Lectures on $n$-dimensional quasiconformal mappings. - Lecture Notes in Mathematics 229, Springer-Verlag, Berlin-Heidelberg-New York, 1971.

[30] Weller, G. P.: Equivalent sizes of Lipschitz manifolds and the smoothing problem. - Osaka J. Math. 10, 1973, 507-510.

[31] Whitehead, J. H. C.: Manifolds with transverse fields in euclidean space. - Ann. of Math. (2) $73,1961,154-212$.

132] Wilker, J. B.: Lipschitz pairs of metric subspaces. - Fund. Math. 71, 1971, 127-130.

\author{
University of Helsinki \\ Department of Mathematics \\ SF-00100 Helsinki 10 \\ Finland
}

Received 15 February 1976 